\title{
Hydrodynamic effective field theory and the analyticity of hydrostatic correlators
}

\author{
Akash Jain, ${ }^{a}$ Pavel Kovtun, ${ }^{a}$ Adam Ritz ${ }^{a}$ and Ashish Shukla ${ }^{b, a}$ \\ ${ }^{a}$ Department of Physics 8 Astronomy, University of Victoria, \\ 3800 Finnerty Road, Victoria, British Columbia V8P 5C2, Canada \\ ${ }^{b}$ Perimeter Institute for Theoretical Physics, \\ 31 Caroline Street North, Waterloo, Ontario, N2L 2Y5, Canada \\ E-mail: ajain@uvic.ca, pkovtun@uvic.ca, aritz@uvic.ca, \\ ashukla@perimeterinstitute.ca
}

ABSTRACT: We study one-loop corrections to retarded and symmetric hydrostatic correlation functions within the Schwinger-Keldysh effective field theory framework for relativistic hydrodynamics, focusing on charge diffusion. We first consider the simplified setup with only diffusive charge density fluctuations, and then augment it with momentum fluctuations in a model where the sound modes can be ignored. We show that the loop corrections, which generically induce non-analyticities and long-range effects at finite frequency, non-trivially preserve analyticity of retarded correlation functions in spatial momentum due to the KMS constraint, as a manifestation of thermal screening. For the purposes of this analysis, we develop an interacting field theory for diffusive hydrodynamics, seen as a limit of relativistic hydrodynamics in the absence of temperature and longitudinal velocity fluctuations.

Keywords: Effective Field Theories, Stochastic Processes, Thermal Field Theory, Quantum Dissipative Systems

ArXiv EPrint: 2011.03691 


\section{Contents}

1 Introduction 1

1.1 Thermal correlators and summary of results 3

2 Density correlation functions in simple diffusion 6

2.1 Simple stochastic diffusion 6

$\begin{array}{lll}2.2 & \text { Linear expansion and interactions } & 7\end{array}$

$\begin{array}{lll}2.3 & \text { One-loop corrections from density fluctuations } & 10\end{array}$

3 Density correlation functions in diffusive hydrodynamics 12

$\begin{array}{lll}3.1 & \text { Stochastic diffusive hydrodynamics } & 12\end{array}$

$\begin{array}{ll}3.2 & \text { Linear expansion and interactions } \\ 3.34\end{array}$

3.3 One-loop corrections from momentum fluctuations 16

$\begin{array}{lll}\text { 3.3.1 Retarded correlation function } & 16\end{array}$

$\begin{array}{lll}\text { 3.3.2 Symmetric correlation function } & 18\end{array}$

$\begin{array}{lll}4 & \text { Discussion } & 19\end{array}$

A The effective action for diffusive hydrodynamics 20

B Loop calculations $\quad 22$

B.1 Computation of $\Gamma_{a r} \quad 23$

B.2 Computation of $\Gamma_{a r}^{\prime} \quad 25$

B.3 Computation of $\Gamma_{a a}$

\section{Introduction}

Hydrodynamics provides an effective description of near-equilibrium thermal states, by focusing on the conserved charges and their transport properties. However, despite its generality, hydrodynamics is incomplete as a true effective field theory (EFT), as it accounts only for dissipation but not the stochastic fluctuations that are present even in equilibrium. Over the past few years, there has been significant progress in our understanding of how to combine the symmetries and the relevant fluctuation degrees of freedom into a complete hydrodynamic EFT, building off the microscopic Schwinger-Keldysh (SK) formalism [1-7] (see [8] for a pedagogical review). Proper consideration of stochastic fluctuations has already led to qualitatively new results in the non-equilibrium (non-hydrostatic) regime. For instance, it was recently discovered that new "stochastic transport coefficients" emerge at higher derivative orders due to stochastic interactions that have no analogue in "classical" hydrodynamics, but nonetheless affect the low-energy behaviour of hydrodynamic correlation functions [9]. EFT tools have also been recently used to revisit long-time tails due to density fluctuations at one-loop order [10]. 
Despite these qualitative departures from classical hydrodynamics, one expects that the EFT results will still boil down to their well-known hydrostatic form as we dial back to equilibrium. In particular, it has been appreciated that careful consideration of the equilibrium limit provides a powerful set of consistency conditions on the hydrodynamic framework $[11,12]$. These conditions restrict the hydrodynamic constitutive relations in a manner that was understood previously to be due only to a local formulation of the second law of thermodynamics. Focusing on relativistic hydrodynamics with a conserved U(1) charge, in a nutshell, one demands that the equilibrium values of the conserved energymomentum tensor $T^{\mu \nu}$ and charge current $J^{\mu}$ can be derived from a hydrostatic generating functional, $\mathcal{W}=T_{0}^{-1} \int d^{d} x \sqrt{-g} \mathcal{F}\left(g_{\mu \nu}, A_{\mu}\right)$, expressed in terms of the background metric $g_{\mu \nu}$ and gauge field sources $A_{\mu}$. Here $T_{0}$ is the constant temperature of the global thermal state and $d$ is the number of spatial dimensions. To wit,

$$
\delta \mathcal{W}\left[g_{\mu \nu}, A_{\mu}\right]=T_{0}^{-1} \int d^{d} x \sqrt{-g}\left(\frac{1}{2} T_{\text {eq }}^{\mu \nu} \delta g_{\mu \nu}+J_{\text {eq }}^{\mu} \delta A_{\mu}\right) .
$$

The associated conservation equations $\nabla_{\mu} T^{\mu \nu}=F^{\nu \lambda} J_{\lambda}$ and $\nabla_{\mu} J^{\mu}=0$ follow directly from the diffeomorphism and gauge invariance of $\mathcal{W}$. An important ingredient in implementing the ensuing constraints on the hydrodynamic constitutive relations is the finiteness of the static correlation length. This feature is expected for generic thermal systems that are not at second order critical points and do not have spontaneously broken symmetries, and implies that retarded hydrostatic (time-independent) correlation functions should fall-off exponentially at large distances. Equivalently, the Fourier space zero-frequency retarded correlation functions must be analytic in an expansion in small spatial momentum. The generating functional thus admits a derivative expansion, and one can obtain the hydrodynamic constitutive relations in the static limit order-by-order in this expansion.

Moving away from thermal equilibrium, the full EFT formalism of hydrodynamics should capture the physics of small fluctuations about the hydrostatic equilibrium state. Thus, hydrodynamic correlation functions should reduce to those derived from the equilibrium generating functional on integrating out all the fluctuation modes. Of particular interest is the requirement of analyticity at small spatial momenta noted above, that follows directly from thermal screening and the finite static correlation length. In the presence of stochastic interactions, it is known that hydrodynamic correlation functions are generically non-analytic out of equilibrium due to the presence of long-time tails [13]. The requirement that these long-distance or infrared non-analyticities must drop out at all loop orders as we specialize to hydrostatic configurations, is quite non-trivial and poses a rigid consistency check for the EFT framework. This question is particularly important because the treelevel propagators for the fluctuation degrees of freedom in these EFTs lack any intrinsic scale that could define the finite spatial correlation length. In fact, the lack of such a scale is precisely what leads to the physics of long-time tails out of equilibrium.

A crucial ingredient in the EFT framework is the discrete Kubo-Martin-Schwinger (KMS) symmetry [4]. It is motivated from its namesake KMS condition in thermal field theory $[14,15]$, and ensures that the non-linear fluctuation-dissipation theorem [16] is satisfied by the EFT framework. While the KMS condition captures the fact that the system 
in question is fluctuating around the thermal state, by itself it does not guarantee the finiteness of the spatial correlation length. However, combined with the structure of hydrodynamic EFTs, we find that the KMS symmetry does, in fact, conspire in a nontrivial way to ensure the analyticity of hydrostatic correlators. The goal of this paper is to illustrate how this analyticity emerges explicitly at one-loop order in relativistic hydrodynamics for density-density two-point functions.

For simplicity, we will work in a kind of "incompressible limit", where sound modes are frozen and the theory only has longitudinal charge diffusion and transverse shear diffusion modes in the low-energy spectrum (see [17] for an early discussion along this direction). Since sound modes are typically "higher energy" compared to diffusive modes, we expect that the simplified model faithfully captures the low-energy behaviour of the EFT. Existing notions of "incompressibility" in the literature, however, are only consistent with linearised hydrodynamics. As we include interactions, due care has to be taken while defining this limit so as to not violate the underlying structure of the EFT, in particular the KMS condition. To reaffirm the role of KMS symmetry in ensuring the analyticity of hydrostatic correlators, for the majority of this paper we will lift the KMS symmetry by untying the dissipative and stochastic fluctuation parameters in the EFT. The simplified model that we develop in this paper, which we dub diffusive hydrodynamics, will also be helpful more generally to probe stochastic signatures in hydrodynamics in the presence of interacting momentum modes. In the remainder of this introductory section, we will elaborate further on the analyticity features of interest, and also summarize our results.

\subsection{Thermal correlators and summary of results}

Recall that for a generic set of bosonic Hermitian operators $\mathcal{O}_{I}$, the retarded and symmetric correlators are defined respectively as

$$
\begin{aligned}
& G_{I J}^{\mathrm{R}}\left(t-t^{\prime}, \mathbf{x}-\mathbf{x}^{\prime}\right) \equiv i \theta\left(t-t^{\prime}\right)\left\langle\left[\mathcal{O}_{I}(t, \mathbf{x}), \mathcal{O}_{J}\left(t^{\prime}, \mathbf{x}^{\prime}\right)\right]\right\rangle, \\
& G_{I J}^{\mathrm{S}}\left(t-t^{\prime}, \mathbf{x}-\mathbf{x}^{\prime}\right) \equiv \frac{1}{2}\left\langle\left\{\mathcal{O}_{I}(t, \mathbf{x}), \mathcal{O}_{J}\left(t^{\prime}, \mathbf{x}^{\prime}\right)\right\}\right\rangle .
\end{aligned}
$$

The thermal expectation values are taken in the grand canonical ensemble with the Hamiltonian $H^{\prime}=H-\mu Q$, where $Q$ is a conserved charge. These correlators are not independent. For example, working in Fourier space and specializing to the hydrodynamic regime $\omega / T_{0} \ll 1$, one can derive the relation

$$
G_{I J}^{\mathrm{S}}(\omega, \boldsymbol{k})=\frac{2 T_{0}}{\omega} \operatorname{Im} G_{I J}^{\mathrm{R}}(\omega, \boldsymbol{k}) .
$$

This is an incarnation of the fluctuation-dissipation theorem, which ties together statistical fluctuations in a thermal system to dissipation, and follows from the KMS periodicity condition in Euclidean time for equilibrium states.

For generic systems in thermal equilibrium, at least away from second order critical points and in the absence of any spontaneously broken symmetries, screening implies a finite static or spatial correlation length. It follows that retarded (or Euclidean) correlation 
functions fall off exponentially at large distances, or equivalently the Fourier space retarded correlators at zero-frequency are analytic at low spatial momentum $\boldsymbol{k}$, i.e.

$$
G_{I J}^{\mathrm{R}}(\omega=0, \boldsymbol{k}) \text { is analytic as } \boldsymbol{k} \rightarrow 0 .
$$

We refer to this condition as spatial analyticity. Importantly, it allows for a well-defined derivative expansion, used previously to develop a hydrostatic generating functional for hydrodynamics $[11,12]$. In the hydrodynamic regime, where $\omega / T_{0} \ll 1,|\boldsymbol{k}| / T_{0} \ll 1$ and we consider perturbations about an equilibrium state, the combination of eqs. (1.3) and (1.4) implies a nontrivial structure for the retarded correlator.

In this paper, we will focus on the correlations of a conserved charge $n=J^{0}$, which is the time-component of a conserved $\mathrm{U}(1)$ current $J^{\mu}$. We can investigate the above features by looking at the symmetric and retarded correlators for $n$. At the tree level in the hydrodynamic EFT (i.e. in the linear response theory), they are known to take the form [18]

$$
\begin{aligned}
& G_{n n}^{\mathrm{R}, \text { tree }}(\omega, \boldsymbol{k})=\frac{i \chi D \boldsymbol{k}^{2}}{\omega+i D \boldsymbol{k}^{2}} \quad \Longrightarrow \quad G_{n n}^{\mathrm{R}, \text { tree }}(\omega \rightarrow 0, \boldsymbol{k})=\chi+\frac{i \chi \omega}{D \boldsymbol{k}^{2}}+\mathcal{O}\left(\omega^{2}\right), \\
& G_{n n}^{\mathrm{S}, \text { tree }}(\omega, \boldsymbol{k})=\frac{2 T \tilde{\sigma} \boldsymbol{k}^{2}}{\omega^{2}+\left(D \boldsymbol{k}^{2}\right)^{2}} \quad \Longrightarrow \quad G_{n n}^{\mathrm{S}, \text { tree }}(\omega \rightarrow 0, \boldsymbol{k})=\frac{2 \tilde{\sigma} T}{D^{2} \boldsymbol{k}^{2}}+\mathcal{O}\left(\omega^{2}\right) .
\end{aligned}
$$

In terms of the chemical potential $\mu$ and conductivity $\sigma$, the coefficients appearing here are the static charge susceptibility $\chi=\partial n /\left.\partial \mu\right|_{T}$ and the diffusion constant $D=\sigma / \chi$. The coefficient $\tilde{\sigma}$ controls the strength of stochastic interactions. In thermal equilibrium, the KMS condition sets $\tilde{\sigma}=\sigma$, leading to these tree-level correlators obeying the fluctuationdissipation theorem in eq. (1.3). We note that the symmetric correlator at $\omega=0$, which is nothing but the tree-level static propagator of $n$, has a pole at $\boldsymbol{k}=0$. This is consistent with our discussion above because symmetric correlators are not restricted by any analyticity requirement. Such poles are, of course, a generic feature of gapless degrees of freedom. The relevant point here is that despite the gapless nature of these tree-level correlations, the retarded correlator is indeed analytic at zero frequency in accordance with eq. (1.4).

While the KMS condition does not play any role at tree-level in ensuring the analyticity of the retarded correlator, it becomes crucial as we start to include loop corrections. In the hydrodynamic EFT, upon accounting for density and transverse-momentum fluctuations within diffusive hydrodynamics, and specializing to $d=3$ spatial dimensions, we find that the correlators in eq. (1.5) admit one-loop corrections that behave at $\omega=0$ as

$$
\begin{aligned}
& G_{n n}^{\mathrm{R}}(\omega=0, \boldsymbol{k})=\chi\left(Z_{\chi}+c_{1}^{\mathrm{R}}|\boldsymbol{k}|+c_{2}^{\mathrm{R}}|\boldsymbol{k}|^{3}+\mathcal{O}\left(\boldsymbol{k}^{4}\right)\right), \\
& G_{n n}^{\mathrm{S}}(\omega=0, \boldsymbol{k})=\frac{2 T \chi}{D \boldsymbol{k}^{2}}\left(Z_{\Lambda}+c_{1}^{\mathrm{S}}|\boldsymbol{k}|+c_{2}^{\mathrm{S}}|\boldsymbol{k}|^{3}+\mathcal{O}\left(\boldsymbol{k}^{4}\right)\right) .
\end{aligned}
$$

Here $Z_{\chi, \Lambda}$ are UV-sensitive renormalization factors with polynomial dependence on the momentum-cutoff of the EFT. These are expected in the low-energy effective field theory due to the irrelevant nature of hydrodynamic interactions. We shall not compute these factors in detail in this paper; a thorough discussion restricted to the scalar diffusion model can be found in [10]. 
The remaining coefficients in eq. (1.7) are interesting physically, because they quantify the infrared and non-analytic corrections to the hydrodynamic correlation functions. These can be explicitly computed in terms of three hydrodynamic parameters: pressure $p(T, \mu)$, shear viscosity $\eta(T, \mu)$, and conductivity $\sigma(T, \mu)$, and two stochastic parameters $\tilde{\eta}(T, \mu)$ and $\tilde{\sigma}(T, \mu)$. The enthalpy density $w(T, \mu)$ and charge density $n(T, \mu)$ are defined using the usual thermodynamic relations $\mathrm{d} p=(w-\mu n) / T \mathrm{~d} T+n \mathrm{~d} \mu$. The bulk viscosity $\zeta(T, \mu)$ and the associated stochastic parameter $\tilde{\zeta}(T, \mu)$ do not appear in the diffusive limit of hydrodynamics. Using the charge susceptibility $\chi=\partial n /\left.\partial \mu\right|_{T}$ and the diffusion constant $D=\sigma / \chi$ from above, and further defining the shear diffusion constant $\gamma_{\eta}=\eta / w$ and the cross-susceptibility $\chi_{\epsilon}=\partial w /\left.\partial \mu\right|_{T}$, we find

$$
\begin{aligned}
c_{1}^{\mathrm{R}}= & \frac{T}{32 \pi D^{2} w}\left(\frac{\tilde{\eta}}{\eta}-\frac{\tilde{\sigma}}{\sigma}\right)\left[\frac{2 \sqrt{\gamma_{\eta} D}\left(D-\gamma_{\eta}\right)}{\left(\gamma_{\eta}+D\right)^{2}}+\arccos \left(\frac{\gamma_{\eta}-D}{\gamma_{\eta}+D}\right)\right], \\
c_{2}^{\mathrm{R}}= & \frac{-T}{32 \pi D \chi}\left[\frac{\partial D}{\partial \mu} \frac{\partial(\tilde{\sigma} / \sigma)}{\partial \mu}+\frac{1}{w} \frac{\partial(\tilde{\eta} / \eta)}{\partial \mu}\left((3 \pi-2) \chi_{\epsilon} D+(7 \pi-20 / 3) n \gamma_{\eta}\right)\right], \\
c_{1}^{\mathrm{S}}= & \frac{T}{32 \pi D^{2} w} \frac{\tilde{\sigma}}{\sigma} \frac{\tilde{\eta}}{\eta}\left[\frac{2 \sqrt{\gamma_{\eta} D}\left(D-\gamma_{\eta}\right)}{\left(\gamma_{\eta}+D\right)^{2}}+\arccos \left(\frac{\gamma_{\eta}-D}{\gamma_{\eta}+D}\right)\right], \\
c_{2}^{\mathrm{S}}= & \frac{-T}{32 \pi D \chi} \frac{\tilde{\sigma}}{\sigma}\left[\frac{\tilde{\sigma}}{\sigma} \frac{1}{D}\left(\frac{\partial D}{\partial \mu}\right)^{2}+\frac{1}{w^{2} \gamma_{\eta}} \frac{\tilde{\eta}}{\eta}\left(\pi \chi_{\epsilon}^{2} D^{2}+(5 \pi-4) n \chi_{\epsilon} D \gamma_{\eta}+2(3 \pi-10 / 3) n^{2} \gamma_{\eta}^{2}\right)\right] \\
& -\frac{T}{32 \pi w^{2} \chi D \gamma_{\eta}} \frac{\tilde{\eta}}{\eta}\left(\frac{\tilde{\eta}}{\eta}-\frac{\tilde{\sigma}}{\sigma}\right)\left(\pi \chi_{\epsilon}^{2} D^{2}-2(3 \pi-10 / 3) n^{2} \gamma_{\eta}^{2}\right) \\
& +\frac{T}{16 \pi w \chi D} \frac{\tilde{\sigma}}{\sigma} \frac{\partial(\tilde{\eta} / \eta)}{\partial \mu}\left((3 \pi-2) \chi_{\epsilon} D+(7 \pi-20 / 3) n \gamma_{\eta}\right) .
\end{aligned}
$$

All the coefficients here are understood to be evaluated on the equilibrium configuration. The explicit functional form of these coefficients is not very important. The important point is that both the retarded and symmetric correlators are generically non-analytic in the static limit. However, upon imposing the KMS condition, which in this case requires setting $\tilde{\sigma}=\sigma$ and $\tilde{\eta}=\eta$, we find

$$
\begin{aligned}
& c_{1}^{\mathrm{R}} \stackrel{\mathrm{KMS}}{=} 0, \quad c_{2}^{\mathrm{R}} \stackrel{\mathrm{KMS}}{=} 0, \\
& c_{1}^{\mathrm{S}} \stackrel{\mathrm{KMS}}{=} \frac{T}{32 \pi D^{2} w}\left[\frac{2 \sqrt{\gamma_{\eta} D}\left(D-\gamma_{\eta}\right)}{\left(\gamma_{\eta}+D\right)^{2}}+\arccos \left(\frac{\gamma_{\eta}-D}{\gamma_{\eta}+D}\right)\right], \\
& c_{2}^{\mathrm{S}} \stackrel{\mathrm{KMS}}{=} \frac{-T}{32 \pi D \chi}\left[\frac{1}{D}\left(\frac{\partial D}{\partial \mu}\right)^{2}+\frac{1}{w^{2} \gamma_{\eta}}\left(\pi \chi_{\epsilon}^{2} D^{2}+(5 \pi-4) n \chi_{\epsilon} D \gamma_{\eta}+2(3 \pi-10 / 3) n^{2} \gamma_{\eta}^{2}\right)\right] .
\end{aligned}
$$

We see that in the presence of the KMS condition, which ensures that the correlators satisfy the fluctuation-dissipation theorem, the non-analytic pieces in the retarded correlator drop out in the hydrostatic limit, reaffirming the consistency of hydrostatic equilibrium in the EFT framework. On the other hand, the symmetric correlator in the hydrostatic limit remains non-analytic. Full analysis of the finite- $\omega$ behaviour of these correlation functions and the ensuing long-time tails will appear in a companion paper [19]. 
The discussion for arbitrary spatial dimensions follows in a similar manner. In $d \neq 1,2$ spatial dimensions, ${ }^{1}$ the corrections inside the brackets in eq. (1.7) typically behave as $|\boldsymbol{k}|^{d-2},|\boldsymbol{k}|^{d}, \ldots$, while in $d=2$ spatial dimensions, they behave as $\log \left(\boldsymbol{k}^{2}\right),|\boldsymbol{k}|^{2} \log \left(\boldsymbol{k}^{2}\right), \ldots$ While we will not derive the explicit form of the associated coefficients in general, we will show in the course of this paper that the one-loop non-analytic corrections to the retarded function drop out in arbitrary dimensions due to the KMS symmetry, maintaining consistency with the existence of thermal equilibrium.

The paper is organized as follows. To sketch out the road-map and develop a qualitative understanding of the results, we will first ignore the momentum modes altogether in section 2, and start with a simplified EFT model of a single diffusive conserved charge as proposed in $[9,10]$. However, unlike the original references, we will lift the KMS symmetry from the model and compute the one-loop corrections to the symmetric and retarded correlation functions directly using the EFT generating functional. Note that we cannot deduce the two correlators using one-another in the absence of KMS symmetry, because the fluctuation-dissipation theorem in eq. (1.3) does not apply. We will then set up the theory of diffusive hydrodynamics in section 3, and repeat the computation of one-loop correlation functions in the presence of transverse momentum fluctuations. We will finish with a discussion in section 4 . In appendix A, we provide further details of the derivation of the EFT for diffusive hydrodynamics and the explicit one-loop computations.

\section{Density correlation functions in simple diffusion}

In this section, we investigate the issue of spatial analyticity of hydrostatic correlators in a simple EFT model with a single diffusive charge, which has all the relevant qualitative features of the full problem. The generalization to full hydrodynamics will be presented in the next section.

\subsection{Simple stochastic diffusion}

Thermal fluctuations are conventionally introduced into the hydrodynamic setup by including stochastic noise sources in the constitutive relations with short-range Gaussian correlations. One way to realize this effective theory is to add a fluctuation field for each conserved hydrodynamic variable in the theory and require that the ensuing effective action, when inserted inside a path integral, reproduces the full set of real-time correlation functions in thermal equilibrium [21-23] (see [18] for a review). Focusing on a single conserved charge $n(\mu)$ with the chemical potential $\mu$, the respective classical conservation equation at one derivative order is given by

$$
\partial_{t} n(\mu)+\partial_{i} J^{i}=0, \quad J^{i}=-\sigma(\mu) \delta^{i j}\left(\partial_{j} \mu-F_{j t}\right),
$$

where $J^{i}$ is the charge flux expressed in terms of $\mu$ and its derivatives. In the following we will often use the "covariant" current $J^{\mu}$ with $J^{0}=n$. Here $F_{i t}=\partial_{i} A_{t}-\partial_{t} A_{i}$ is the

\footnotetext{
${ }^{1}$ Note that in $d=1$ spatial dimension, the leading correction to the retarded correlator goes as $1 /|\boldsymbol{k}|$ and is relevant, signaling the fact that the hydrodynamic description does not apply [20].
} 
electric field associated with the background gauge field $A_{\mu}$ coupled to $J^{\mu}$. The charge conductivity $\sigma(\mu)$ is an arbitrary function of $\mu$, required to be non-negative by the second law of thermodynamics. The equilibrium state is given by $\mu=\mu_{0}$ being a constant.

In the EFT language, the chemical potential $\mu$ is related to the fundamental "chemical shift" field $\Lambda_{\beta}$ as $\mu=T_{0} \Lambda_{\beta}+A_{r t}$, where $A_{r \mu}$ is one of the Schwinger-Keldysh background gauge fields along with $A_{a \mu} .{ }^{2}$ To account for stochastic fluctuations, we add an auxiliary field $\varphi_{a}$ to partner the hydrodynamic field $\Lambda_{\beta}$. The effective action $S$ of the theory is fixed so that it reproduces all the two-point response functions at tree-level as predicted by classical linearized hydrodynamics. Introducing the effective Lagrangian density through $S=\int d^{d+1} x \mathcal{L}$, we have $[4,9]$

$$
\mathcal{L}=J^{\mu}\left(\partial_{\mu} \varphi_{a}+A_{a \mu}\right)+i T_{0} \tilde{\sigma}(\mu)\left(\partial_{i} \varphi_{a}+A_{a i}\right)\left(\partial^{i} \varphi_{a}+A_{a}^{i}\right) .
$$

Here $T_{0}$ is the constant temperature of the thermal equilibrium state. We have introduced an arbitrary coefficient $\tilde{\sigma}(\mu)$ that characterizes the strength of stochastic fluctuations. This is the most general Lagrangian consistent with the Schwinger-Keldysh EFT framework for a single diffusive charge at one-derivative order. However, for this effective Lagrangian to correctly reproduce the hydrodynamic response functions, we need to impose the constraint from the fluctuation-dissipation theorem. This equates the strength of stochastic fluctuations $\tilde{\sigma}$ to the dissipative transport coefficient $\sigma$ (see [18] for more details),

$$
\text { KMS : } \quad \tilde{\sigma}=\sigma .
$$

Assuming the underlying microscopic theory to be CPT-invariant, in the SchwingerKeldysh (SK) effective field theory framework of [4], this follows as a consequence of the dynamical KMS symmetry of the effective action

$$
\begin{gathered}
\mu_{0} \rightarrow-\mu_{0}, \quad \Lambda_{\beta}(x) \rightarrow-\Lambda_{\beta}(-x), \quad \varphi_{a}(x) \rightarrow \varphi_{a}(-x)+i \Lambda_{\beta}(-x), \\
A_{r \mu}(x) \rightarrow-A_{r \mu}(-x), \quad A_{a \mu}(x) \rightarrow-A_{a \mu}(-x)-i T_{0}^{-1}\left(\partial_{t} A_{r \mu}\right)(-x) .
\end{gathered}
$$

In addition, it implies the charge conjugation properties for the coefficients $n(-\mu)=-n(\mu)$, $\sigma(-\mu)=\sigma(\mu)$, and $\tilde{\sigma}(-\mu)=\tilde{\sigma}(\mu)$. In the following, we find that the KMS condition is critical for ensuring the analyticity of retarded correlators in spatial momentum. Hence, to keep track of the non-analytic behaviour explicitly, we will perform the majority of forthcoming manipulations assuming $\tilde{\sigma}$ and $\sigma$ to be independent.

\subsection{Linear expansion and interactions}

To be able to use this theory in a loop expansion, we need to expand the Lagrangian (2.2) order-by-order in fluctuations about the equilibrium state $\Lambda_{\beta}=\mu_{0} / T_{0}, \varphi_{a}=0$; see [9] for details. It is practically easier to work with the fluctuations in the density $\delta n=$ $n\left(T_{0} \Lambda_{\beta}\right)-n\left(\mu_{0}\right)$ rather than $\Lambda_{\beta}$. Ignoring the background fields at first, and ignoring

\footnotetext{
${ }^{2}$ In the EFT language of [4], the true fundamental degree of freedom is the phase field $\varphi_{r}$, which is related to $\Lambda_{\beta}$ above via the definition $\Lambda_{\beta} T_{0}=\mu_{0}+\partial_{t} \varphi_{r}$. For an ordinary fluid phase where the U(1) symmetry is not spontaneously broken, the system respects a spatial chemical shift symmetry: $\varphi_{r}(x) \rightarrow \varphi_{r}(x)-\lambda(\boldsymbol{x})$, and all the shift-invariant information in $\varphi_{r}$ is contained in $\Lambda_{\beta}$.
} 
certain total-derivative terms, we find the "free" Gaussian part of the effective Lagrangian to be

$$
\mathcal{L}_{\text {free }}=-\varphi_{a}\left(\partial_{t} \delta n-D \nabla^{2} \delta n\right)+i T \tilde{\sigma} \partial^{i} \varphi_{a} \partial_{i} \varphi_{a}
$$

where $D=\sigma / \chi$ is the diffusion constant, with $\chi=n^{\prime}(\mu)$ being the charge susceptibility, and $\nabla^{2} \equiv \partial^{i} \partial_{i}$. We have dropped the subscript " 0 " for clarity and the coefficients are understood to be evaluated at equilibrium, i.e. $\mu=\mu_{0}$. This leads to the tree-level propagators

$$
\begin{aligned}
\left\langle\delta n(p) \varphi_{a}(-p)\right\rangle_{0} & =\frac{1}{\omega+i D \boldsymbol{k}^{2}}, \\
\left\langle\varphi_{a}(p) \delta n(-p)\right\rangle_{0} & =\frac{-1}{\omega-i D \boldsymbol{k}^{2}}, \\
\langle\delta n(p) \delta n(-p)\rangle_{0} & =\frac{2 T \tilde{\sigma} \boldsymbol{k}^{2}}{\omega^{2}+D^{2} \boldsymbol{k}^{4}}=\frac{i T \chi \tilde{\sigma} / \sigma}{\omega+i D \boldsymbol{k}^{2}}-\frac{i T \chi \tilde{\sigma} / \sigma}{\omega-i D \boldsymbol{k}^{2}}, \\
\left\langle\varphi_{a}(p) \varphi_{a}(-p)\right\rangle_{0} & =0 .
\end{aligned}
$$

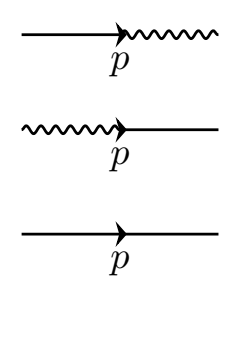

Here $p=(\omega, \boldsymbol{k})$ collectively denotes frequency and momentum. We have denoted $\delta n$ by solid and $\varphi_{a}$ by wavy lines. Note that the $\left\langle\delta n \varphi_{a}\right\rangle$ propagator is purely retarded, $\left\langle\varphi_{a} \delta n\right\rangle$ propagator is purely advanced, while $\langle\delta n \delta n\rangle$ propagator can be expressed as a sum of retarded and advanced pieces. This observation generalizes to full hydrodynamics, and will be important in the explicit loop calculations in the following discussion.

The free diffusive action (2.5) is well-known in the literature (see e.g. [18]), however, it does not account for interactions. To this end, we can expand eq. (2.2) to the next order in $\delta n$ and $\varphi_{a}$ to obtain ${ }^{3}$

$$
\mathcal{L}_{\mathrm{int}}^{3 \mathrm{pt}}=\frac{1}{2} \lambda \delta n^{2} \nabla^{2} \varphi_{a}+i \chi T \tilde{\lambda} \delta n \partial_{i} \varphi_{a} \partial^{i} \varphi_{a}
$$

where the two coupling constants $\lambda$ and $\tilde{\lambda}$ are defined via

$$
\lambda=\frac{1}{\chi} \frac{\partial D}{\partial \mu}, \quad \tilde{\lambda}=\frac{1}{\chi^{2}} \frac{\partial \tilde{\sigma}}{\partial \mu} .
$$

We can work-out the momentum-space Feynman rules for the associated vertices
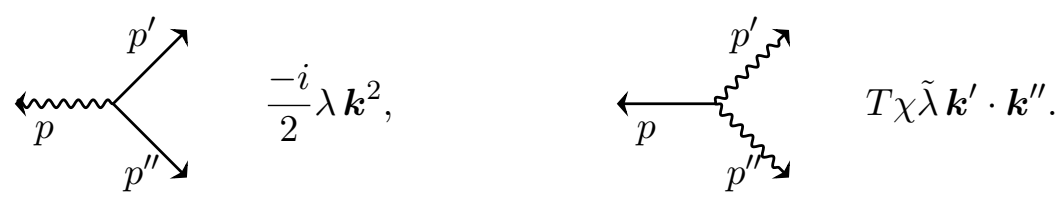

The same procedure can be iterated to arbitrarily high orders in fluctuations, depending on the sensitivity required. This form of the effective action, albeit for energy diffusion instead of charge diffusion, was derived recently in [10].

To be able to compute the correlations functions using the non-equilibrium generating functional [4], we also need the structure of the background field couplings in the EFT.

\footnotetext{
${ }^{3}$ See $[24,25]$ for discussions along similar lines, involving the dependence of transport parameters on the fluctuating fields.
} 
Since, in this work, we are only interested in density correlators, we only turn on the $A_{r t}$, $A_{a t}$ components of the gauge fields. We directly borrow the results from [9], leading to

$$
\begin{aligned}
\mathcal{L}_{\text {source }}^{2 \mathrm{pt}}= & \delta n A_{a t}+\chi \partial_{t} \varphi_{a} A_{r t}+\chi A_{r t} A_{a t}, \\
\mathcal{L}_{\text {source }}^{3 \mathrm{pt}}= & \frac{\chi^{\prime}}{\chi} \delta n A_{r t} A_{a t}+\frac{1}{2} \chi^{\prime} \partial_{t} \varphi_{a} A_{r t}^{2}+\frac{1}{2} \chi^{\prime} A_{r t}^{2} A_{a t} \\
& \quad+\left(\frac{\chi^{\prime}}{\chi} \delta n \partial_{t} \varphi_{a}-\frac{1}{\chi} \frac{\partial \sigma}{\partial \mu} \partial^{i} \delta n \partial_{i} \varphi_{a}+i T \frac{\partial \tilde{\sigma}}{\partial \mu} \partial_{i} \varphi_{a} \partial^{i} \varphi_{a}\right) A_{r t},
\end{aligned}
$$

where $\chi^{\prime}=\partial \chi / \partial \mu$. Denoting $A_{r t}$ by a dotted and $i A_{a t}$ by a dashed line, we can represent these diagrammatically as

$$
\begin{aligned}
& \vdash p \quad 1, \\
& \text {, }
\end{aligned}
$$

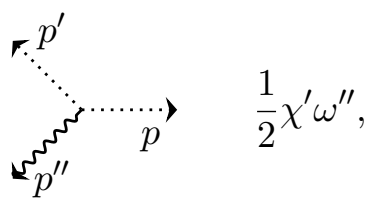

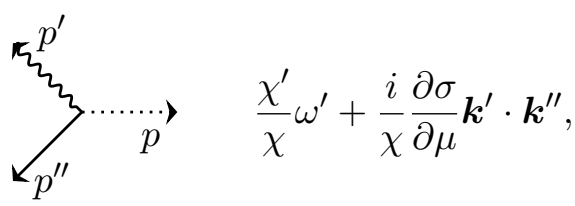

$$
\begin{aligned}
& \operatorname{mon}_{p} \ldots \ldots \ldots \\
& \overbrace{p^{\prime \prime}}^{p^{\prime}} \stackrel{\chi^{\prime}}{y^{\prime}},
\end{aligned}
$$

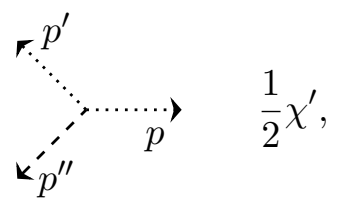

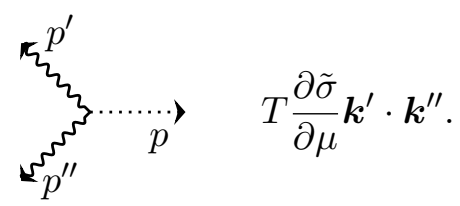

The first two of these are the usual linear couplings between operators and sources in quantum field theories, while the remaining are non-linear couplings. Frequency and momenta going away from the vertex are taken to be positive.

Let us briefly comment on the dimension counting scheme that we implement in the following discussion. For diffusive processes, it is typically argued that $\partial_{t} \sim \partial^{i} \partial_{i}$. Following e.g. [26], we can demand that $\left[\partial_{i}\right]=1$ and require that the couplings in spatial kinetic terms in eq. (2.5) are dimensionless. If we take $\left[\partial_{t}\right]=2$ to ensure consistent scaling, and thus $[\mathcal{L}]=d+2$, we find $\left[\varphi_{a}\right]=[\delta n]=d / 2$, and it follows that $[\lambda]=[\tilde{\lambda}]=-d / 2$ and thus both interactions in eq. (2.7) are irrelevant. We therefore expect that loops will induce power-like sensitivity to the UV cutoff. To count loops $(L)$, we can use the relation $L=1+(N / 2-1) V-E / 2$ for $N^{t h}$ order vertices in diagrams with $E$ external lines. In the case of interest below, $E=2$, and so at one-loop order, $V=2 /(N-2)$. Thus, one-loop corrections require diagrams with 2 cubic vertices. In principle, diagrams with a single quartic vertex can also contribute at this order, however the contributions from such diagrams to the two-point functions are purely UV-cutoff dependent and only renormalize the classical hydrodynamic parameters $[9,10]$. 


\subsection{One-loop corrections from density fluctuations}

Defining the Schwinger-Keldysh generating functional as $[4]^{4}$

$$
\exp W\left[A_{r \mu}, A_{a \mu}\right]=\int \mathcal{D} \delta n \mathcal{D} \varphi_{a} \exp \left(i \int \mathrm{d}^{d+1} x \mathcal{L}\right),
$$

we can compute the retarded and symmetric correlators of $n$ via

$$
\begin{aligned}
& G_{n n}^{\mathrm{R}}(p)=\frac{-i \delta^{2} W}{\delta A_{a t}(p) \delta A_{r t}(-p)}=\chi-\omega \chi\left\langle\delta n(p) \varphi_{a}(-p)\right\rangle+(\text { source coupling diagrams }), \\
& G_{n n}^{\mathrm{S}}(p)=\frac{-\delta^{2} W}{\delta A_{a t}(p) \delta A_{a t}(-p)}=\langle\delta n(p) \delta n(-p)\rangle .
\end{aligned}
$$

Using the propagators in eq. (2.6), it is trivial to check that we reproduce the tree-level results for the two correlation functions given in eq. (1.5).

We will now compute the stochastic loop corrections to the classical tree-level propagators in eq. (1.5). This amounts to integrating out the stochastic noise field $\varphi_{a}$ at one-loop order using the interaction vertices defined above in eqs. (2.7) and (2.10). Let us start with the retarded function. At one-loop order, we have two diagrams contributing to the $\left\langle\delta n \varphi_{a}\right\rangle$ propagator (see [9] for details)
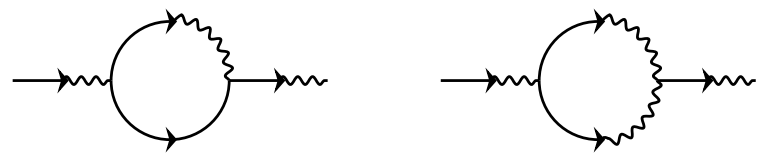

In addition, we have two diagrams involving non-linear background source couplings
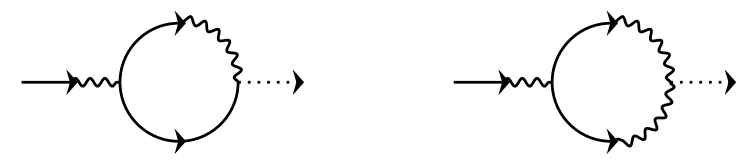

We can compute these diagrams to find the one-loop correction to the retarded density correlator; see appendix B for calculational details. We find the one-loop correction to the retarded correlator to be

$$
G_{n n}^{\mathrm{R}, 1-\operatorname{loop}}(p)=\frac{i T \lambda \boldsymbol{k}^{2}}{\left(\omega+i D \boldsymbol{k}^{2}\right)^{2}}\left(\frac{\tilde{\sigma}}{\sigma} \omega \chi^{2} \lambda-\left(\omega+i D \boldsymbol{k}^{2}\right) \sigma \frac{\partial(\tilde{\sigma} / \sigma)}{\partial \mu}\right) \mathcal{I}_{1}(p)
$$

where $p=(\omega, \boldsymbol{k})$ and $\mathcal{I}_{1}(p)$ is the result of the loop momentum integral

$$
\begin{aligned}
\mathcal{I}_{1}(p)=\frac{\boldsymbol{k}^{2}}{2} \int^{\Lambda} \frac{\mathrm{d}^{d} \boldsymbol{k}^{\prime}}{(2 \pi)^{d}} \frac{1}{i \omega-D\left(\boldsymbol{k}^{2}+\left(\boldsymbol{k}-\boldsymbol{k}^{\prime}\right)^{2}\right)} & \stackrel{d=3}{=} \frac{\boldsymbol{k}^{2}}{32 \pi D} \sqrt{\boldsymbol{k}^{2}-\frac{2 i \omega}{D}}+\mathcal{O}(\Lambda), \\
& \stackrel{d=2}{=} \frac{\boldsymbol{k}^{2}}{16 \pi D} \ln \left(\boldsymbol{k}^{2}-\frac{2 i \omega}{D}\right)+\mathcal{O}(\ln \Lambda) .
\end{aligned}
$$

\footnotetext{
${ }^{4}$ Technically, the EFT path integral should be performed over the phase field $\varphi_{r}$, with the field space volume divided by the associated spatial chemical shift symmetry; see footnote 2 . Passing over to $\delta n$ or $\Lambda_{\beta}$ might generically result in additional Jacobian factors that we shall ignore for now.
} 
The value of this integral depends on the UV-cutoff $\Lambda$ and the number of spatial dimensions $d$. Above, we have explicitly computed it for $d=2,3$ for reference. We see that, generically, the one-loop corrections at finite frequency exhibit IR-sensitive non-analytic dependence on $\omega$, reflecting the physics of long-time tails, which invalidates the hydrodynamic derivative expansion at a given order [27]. However, if we restrict to the hydrostatic $(\omega=0)$ limit, we have argued that thermal screening should render the correlator analytic. Setting $\omega=0$, the one-loop correction reduces to

$$
G_{n n}^{\mathrm{R}, 1-\mathrm{loop}}(0, \boldsymbol{k})=-T \chi \lambda \frac{\partial(\tilde{\sigma} / \sigma)}{\partial \mu} \mathcal{I}_{1}(0, \boldsymbol{k}) \stackrel{\mathrm{KMS}}{=} 0,
$$

which vanishes trivially upon imposing the KMS condition $\tilde{\sigma}=\sigma$, irrespective of the dimensionality. Hence, we see that the KMS condition is crucial to reproduce the analyticity of retarded correlators in the hydrostatic limit.

We can repeat the same procedure for the symmetric correlator. In this case, we do not have any background coupling diagrams at one-loop order. We only need to consider the seven diagrams correcting the $\langle\delta n \delta n\rangle$ propagator
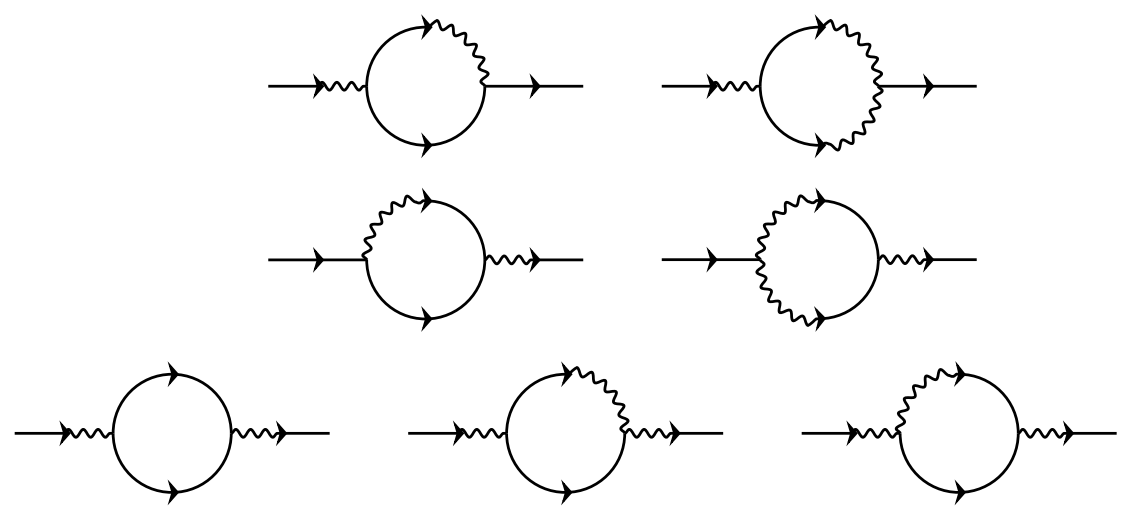

Relegating the explicit computations to the appendix, we find that

$$
G_{n n}^{\text {S,1-loop }}(p)=\frac{T^{2} \chi^{2} \lambda^{2} \boldsymbol{k}^{2}}{\left(\omega+i D \boldsymbol{k}^{2}\right)^{2}} \frac{\tilde{\sigma}^{2}}{\sigma^{2}} \mathcal{I}_{1}(p)+\frac{T^{2} \chi^{2} \lambda^{2} \boldsymbol{k}^{2}}{\left(\omega-i D \boldsymbol{k}^{2}\right)^{2}} \frac{\tilde{\sigma}^{2}}{\sigma^{2}} \mathcal{I}_{1}(-p) .
$$

It can be explicitly checked that, on imposing the KMS condition, these corrections satisfy the fluctuation-dissipation theorem (1.3) along with the retarded correlator corrections in eq. (2.15). Finally, in the $\omega=0$ limit, the symmetric correlator behaves as

$$
G_{n n}^{\mathrm{S}, 1-\operatorname{loop}}(0, \boldsymbol{k})=\frac{-2 T^{2} \chi^{2} \lambda^{2}}{D^{2} \boldsymbol{k}^{2}} \frac{\tilde{\sigma}^{2}}{\sigma^{2}} \mathcal{I}_{1}(0, \boldsymbol{k}),
$$

which survives the KMS limit $\tilde{\sigma}=\sigma$. This indicates that IR-singular non-analytic terms are generated in the hydrostatic limit, as a manifestation of the lack of any intrinsic mass scale in the tree-level propagators (2.6). This may appear surprising, given the earlier discussion of thermal screening. However, the symmetric correlator is not determined explicitly by the hydrostatic generating functional and does not have to meet the analyticity requirement. The leading IR-singularity, proportional to $1 / \boldsymbol{k}^{2}$, to the symmetric correlator is still given by the tree-level propagator. Also, note that the one-loop correction to the symmetric correlator in the static limit does not depend upon $\tilde{\lambda}$. 


\section{Density correlation functions in diffusive hydrodynamics}

The discussion of charge diffusion above can be extended to the full theory of relativistic hydrodynamics. We will again focus on the transport of a single conserved charge, but consistently incorporate additional hydrodynamic momentum modes in the thermal bath. We will develop an effective field theory for diffusive hydrodynamics, where the sound modes have been frozen and the theory only contains diffusive charge and shear modes. This allows us to compute the hydrodynamic correlators in a slightly simpler setting, while still incorporating the non-trivial effects of momentum modes. Our goal is not to provide a comprehensive analysis, but to test the spatial analyticity of retarded charge density correlators in a system with additional gapless modes, specifically those associated with momentum fluctuations.

\subsection{Stochastic diffusive hydrodynamics}

The classical dynamical equations for relativistic hydrodynamics are given by charge and energy-momentum conservation,

$$
\partial_{\mu} J^{\mu}=0, \quad \partial_{\mu} T^{\mu \nu}=F^{\nu \lambda} J_{\lambda}
$$

along with the constitutive relations expressing $J^{\mu}$ and $T^{\mu \nu}$ in terms of the hydrodynamic variables: temperature $T$, chemical potential $\mu$, fluid four-velocity $u^{\mu}$, and their derivatives. For instance, in the Landau frame up to first order in derivatives we have ${ }^{5}$

$$
\begin{aligned}
J^{\mu}= & n(T, \mu) u^{\mu}-\sigma(T, \mu) \Delta^{\mu \lambda}\left(T \partial_{\lambda}\left(\frac{\mu}{T}\right)-F_{\lambda \nu} u^{\nu}\right), \\
T^{\mu \nu}= & \epsilon(T, \mu) u^{\mu} u^{\nu}+p(T, \mu) \Delta^{\mu \nu} \\
& -2 \eta(T, \mu)\left(\Delta^{\rho(\mu} \Delta^{\nu) \sigma} \partial_{\rho} u_{\sigma}-\frac{1}{d} \Delta^{\mu \nu} \partial_{\lambda} u^{\lambda}\right)-\zeta(T, \mu) \Delta^{\mu \nu} \partial_{\lambda} u^{\lambda} .
\end{aligned}
$$

We have introduced the background gauge field $A_{\mu}$ coupled to $J^{\mu}$, and the associated field strength $F_{\mu \nu}=2 \partial_{[\mu} A_{\nu]}$, but have avoided introducing the metric source for $T^{\mu \nu}$ that we do not require for our purposes. Here $\Delta^{\mu \nu}=\eta^{\mu \nu}+u^{\mu} u^{\nu}$ is the projector transverse to the fluid velocity. The thermodynamic pressure $p$, energy density $\epsilon$, and charge density $n$ are functions of $T$ and $\mu$, and related to each other via the thermodynamic relations $d p=s d T+n d \mu$ and $\epsilon+p=T s+\mu n$ for some entropy density $s$. The charge conductivity $\sigma$, shear viscosity $\eta$, and bulk viscosity $\zeta$ are non-negative functions of $T$ and $\mu$. We shall be interested in fluctuations about an equilibrium state with $\mu=\mu_{0}$ at a constant global temperature $T=T_{0}$ in the rest frame $u^{\mu}=(1, \mathbf{0})$.

The EFT for hydrodynamics is formulated in terms of a thermal vector $\beta^{\mu}$ and "chemical shift" field $\Lambda_{\beta}$, along with their stochastic noise partners $X_{a}^{\mu}$ and $\varphi_{a}$. These are related to the aforementioned hydrodynamic fields as $u^{\mu} / T=\beta^{\mu}$ and $\mu / T=\Lambda_{\beta}+\beta^{\mu} A_{r \mu}$. Similar to the diffusive EFT, we introduce the Schwinger-Keldysh double copies of background

\footnotetext{
${ }^{5}$ The symmetrization of indices follows the convention $\mathrm{A}^{(\mu} \mathrm{B}^{\nu)}=\frac{1}{2}\left(\mathrm{~A}^{\mu} \mathrm{B}^{\nu}+\mathrm{A}^{\nu} \mathrm{B}^{\mu}\right)$. Similarly, antisymmetrization follows the convention $\mathrm{A}^{[\mu} \mathrm{B}^{\nu]}=\frac{1}{2}\left(\mathrm{~A}^{\mu} \mathrm{B}^{\nu}-\mathrm{A}^{\nu} \mathrm{B}^{\mu}\right)$.
} 
gauge fields $A_{r \mu}, A_{a \mu}$. With all the ingredients in place, the full non-linear effective action for relativistic hydrodynamics takes the form [2]

$$
\begin{aligned}
\mathcal{L}= & J^{\mu}\left(\partial_{\mu} \varphi_{a}+A_{a \mu}+£_{X_{a}} A_{r \mu}\right)+i T \tilde{\sigma} \Delta^{\mu \nu}\left(\partial_{\mu} \varphi_{a}+A_{a \mu}+£_{X_{a}} A_{r \mu}\right)\left(\partial_{\nu} \varphi_{a}+A_{a \nu}+£_{X_{a}} A_{r \nu}\right) \\
& +T^{\mu \nu} \partial_{\mu} X_{a \nu}+i T\left(2 \tilde{\eta} \Delta^{\mu(\rho} \Delta^{\sigma) \nu}+\left(\tilde{\zeta}-\frac{2}{d} \tilde{\eta}\right) \Delta^{\mu \nu} \Delta^{\rho \sigma}\right) \partial_{\mu} X_{a \nu} \partial_{\rho} X_{a \sigma}+\cdots,
\end{aligned}
$$

where $£_{X_{a}}$ denotes a Lie derivative along $X_{a}^{\mu}$. We have introduced the stochastic coefficients $\tilde{\sigma}, \tilde{\eta}$, and $\tilde{\zeta}$, which are also arbitrary non-negative functions of $T$ and $\mu$. We can recover the previous diffusive model in eq. (2.2) by simply setting $X_{a}^{\mu}=0$ and $u^{\mu} / T=\delta_{t}^{\mu} / T_{0}$. This is the most general effective action for relativistic hydrodynamics, in Landau frame, compatible with the Schwinger-Keldysh framework of [4], truncated at one-derivative order. We utilize the usual derivative counting scheme for relativistic hydrodynamics: in the " $r$ " sector we take $u^{\mu}, T, \mu, A_{r \mu} \sim \mathcal{O}\left(\partial^{0}\right)$, while in the " $a$ " sector we have $X_{a}^{\mu}, \varphi_{a} \sim \mathcal{O}\left(\partial^{0}\right)$, $A_{a \mu} \sim \mathcal{O}\left(\partial^{1}\right)$.

The dynamical KMS symmetry in eq. (2.4) generalizes to a covariant version,

$$
\begin{gathered}
\mu_{0} \rightarrow-\mu_{0}, \quad \beta^{\mu}(x) \rightarrow \beta^{\mu}(-x), \quad \Lambda_{\beta}(x) \rightarrow-\Lambda_{\beta}(-x), \\
\varphi_{a}(x) \rightarrow \varphi_{a}(-x)+i \Lambda_{\beta}(-x), \quad X_{a}^{\mu}(x) \rightarrow-X_{a}^{\mu}(-x)-i\left(\beta^{\mu}(-x)-T_{0}^{-1} \delta_{t}^{\mu}\right), \\
A_{r \mu}(x) \rightarrow-A_{r \mu}(-x), \quad A_{a \mu}(x) \rightarrow-A_{a \mu}(-x)-i\left(£_{\beta} A_{r \mu}\right)(-x),
\end{gathered}
$$

where $£_{\beta}$ denotes a Lie-derivative along $\beta^{\mu}$. In addition to the charge conjugation properties of various coefficients, the KMS symmetry relates the stochastic fluctuation coefficients to the dissipative transport coefficients via

$$
\text { KMS : } \quad \tilde{\sigma}=\sigma, \quad \tilde{\eta}=\eta, \quad \tilde{\zeta}=\zeta .
$$

However, we shall not implement these constraints for now to investigate the role of KMS in ensuring the analyticity of hydrostatic retarded correlators.

Similar to our discussion in section 2, we can expand the Lagrangian order-by-order in the hydrodynamic fluctuations $\delta \beta^{\mu}=\beta^{\mu}-\delta_{t}^{\mu} / T_{0}, \delta \Lambda_{\beta}=\Lambda_{\beta}-\mu_{0} / T_{0}$, and associated noise $X_{a}^{\mu}, \varphi_{a}$. The full theory turns out to be quite complicated in practice. To make our lives simpler, we will focus on a sub-sector, which we call diffusive hydrodynamics, where the temperature and the longitudinal velocity fluctuations have been frozen and so can be ignored. This is similar to the well-known "incompressible limit" of non-relativistic hydrodynamics and amounts to ignoring the sound modes and only focusing on the diffusive modes. We take

$$
\delta \beta^{t}=0, \quad \partial_{i} \delta \beta^{i}=0, \quad X_{a}^{t}=0, \quad \partial_{i} X_{a}^{i}=0 .
$$

The last two conditions are required for consistency with the KMS condition (3.5). This removes the energy conservation equation and the trace of the spatial stress tensor from the effective action. In terms of the original hydrodynamic variables, the first two conditions imply ${ }^{6}$

$$
\delta T \equiv T-T_{0}=\frac{T_{0}}{2} \boldsymbol{u}^{2}+\ldots, \quad \partial_{i} u^{i}=0+\ldots,
$$

\footnotetext{
${ }^{6}$ If we were to decompose the relativistic fluid velocity according to $u^{\mu}=(1, \boldsymbol{v}) / \sqrt{1-\boldsymbol{v}^{2}}$, these conditions will imply $\partial_{i} v^{i}=0$ exactly, making contact with the incompressible limit of hydrodynamics.
} 
where $\boldsymbol{u}^{2}=u^{i} u_{i}$. We have suppressed terms that are cubic or higher order in $\delta \beta^{i}$. To impose these conditions in the EFT, we include additional constraint terms in the effective action

$$
\mathcal{L}_{\text {fixing }}=\Psi_{a} \partial_{i} \delta \beta^{i}+\Psi_{r} \partial_{i} X_{a}^{i}
$$

for arbitrary Lagrange multipliers $\Psi_{r}, \Psi_{a}$. For consistency with the KMS condition (3.5), these multipliers must transform as

$$
\Psi_{r}(x) \rightarrow \Psi_{r}(-x), \quad \Psi_{a}(x) \rightarrow-\Psi_{a}(-x)+i \Psi_{r}(-x)
$$

\subsection{Linear expansion and interactions}

It turns out to be more convenient to work with density fluctuations $\delta n=\left.J^{t}\right|_{A_{r}=0}-n\left(\mu_{0}\right)+$ $2 i T \tilde{\sigma} u^{i} \partial_{i} \varphi_{a}$ and momentum fluctuations $\pi^{i}=T^{t i}+2 i T \tilde{\eta}\left(u^{k} \partial_{k} X_{a}^{i}+u_{k} \partial^{i} X_{a}^{k}\right) .{ }^{7}$ The free part of the effective Lagrangian (3.4), along with the Lagrange multiplier terms, takes the form

$$
\begin{aligned}
\mathcal{L}_{\text {free }}= & -\varphi_{a}\left(\partial_{t}-D \nabla^{2}\right) \delta n+i T \tilde{\sigma} \partial_{i} \varphi_{a} \partial^{i} \varphi_{a}-X_{a}^{i}\left(\partial_{t}-\gamma_{\eta} \nabla^{2}\right) \pi_{i}+i T \tilde{\eta} \partial_{k} X_{a}^{j} \partial^{k} X_{a j} \\
& +\frac{1}{T w} \Psi_{a} \partial_{i} \pi^{i}+\Psi_{r} \partial_{i} X_{a}^{i} .
\end{aligned}
$$

The explicit calculational details can be found in appendix A. As before, $D=\sigma / \chi$ is the charge diffusion constant with $\chi=\partial n /\left.\partial \mu\right|_{T}$ being the charge susceptibility, while $\gamma_{\eta}=\eta / w$ is the shear diffusion constant with $w=\epsilon+p$ being the enthalpy density. We still have the same tree-level propagators in the charge sector, eq. (2.6), but we also find new momentum dependent propagators given by

$$
\begin{aligned}
& \left\langle\pi^{i}(p) X_{a}^{j}(-p)\right\rangle_{0}=\left(\delta^{i j}-\frac{k^{i} k^{j}}{\boldsymbol{k}^{2}}\right) \frac{1}{\omega+i \gamma_{\eta} \boldsymbol{k}^{2}}, \\
& \left\langle X_{a}^{i}(p) \pi^{j}(-p)\right\rangle_{0}=\left(\delta^{i j}-\frac{k^{i} k^{j}}{\boldsymbol{k}^{2}}\right) \frac{-1}{\omega-i \gamma_{\eta} \boldsymbol{k}^{2}}, \\
& \left\langle\pi^{i}(p) \pi^{j}(-p)\right\rangle_{0}=\left(\delta^{i j}-\frac{k^{i} k^{j}}{\boldsymbol{k}^{2}}\right) \frac{2 T \tilde{\eta} \boldsymbol{k}^{2}}{\omega^{2}+\gamma_{\eta}^{2} \boldsymbol{k}^{4}}, \\
& \left\langle X_{a}^{i}(p) X_{a}^{j}(-p)\right\rangle_{0}=0 .
\end{aligned}
$$
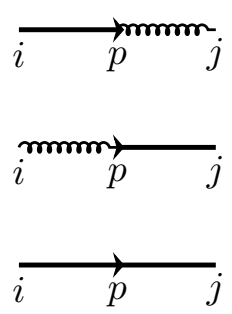

We have denoted $\pi^{i}$ with a bold and $X_{a}^{i}$ with a coiled line. All the cross-propagators between the momentum and the charge sectors are zero. This is a feature of the simplified diffusive hydrodynamic theory eq. (3.7), and will not be the case in full hydrodynamics in the presence of sound modes. These propagators are made transverse to momentum by the presence of the Lagrange multipliers. The propagators for the multipliers themselves can be computed, but they do not have any physical poles and drop out for our case of interest.

\footnotetext{
${ }^{7}$ These definitions of $\delta n$ and $\pi^{i}$, including the imaginary parts, are the true charge and momentum densities that are conserved in the presence of stochastic fluctuations, unlike $J^{0}$ and $T^{0 i}$ that are only conserved classically; see appendix A for details.
} 
At the next order in fluctuations, we have the three-point interaction Lagrangian

$$
\begin{aligned}
\mathcal{L}_{\mathrm{int}}^{3 \mathrm{pt}}= & \frac{1}{2} \lambda \delta n^{2} \nabla^{2} \varphi_{a}+i T \chi \tilde{\lambda} \delta n \partial_{i} \varphi_{a} \partial^{i} \varphi_{a}+\frac{1}{w} \pi^{i} \delta n \partial_{i} \varphi_{a}+\frac{1}{w} \pi^{i} \pi^{j} \partial_{i} X_{a j} \\
& +\frac{1}{2} \lambda_{\pi} \pi^{2} \nabla^{2} \varphi_{a}-\psi \pi^{j} \pi^{i} \partial_{i} \partial_{j} \varphi_{a}-i T w \tilde{\psi}\left(\pi_{j} \partial^{i} X_{a}^{j}+\pi_{j} \partial^{j} X_{a}^{i}\right) \partial_{i} \varphi_{a} \\
& -\gamma \delta n \pi^{j} \nabla^{2} X_{a j}-2 \theta \delta n \partial^{(i} \pi^{j)} \partial_{i} X_{a j}+2 i T w \tilde{\theta} \delta n \partial^{(i} X_{a}^{j)} \partial_{i} X_{a j} \\
& -\frac{\chi \epsilon}{T \chi w^{2}} \Psi_{a} \pi^{i} \partial_{i} \delta n,
\end{aligned}
$$

which generalizes eq. (2.7). Various coupling constants are defined as

$$
\begin{gathered}
\chi_{\epsilon}=\frac{\partial w}{\partial \mu}, \quad \lambda=\frac{1}{\chi} \frac{\partial D}{\partial \mu}, \quad \tilde{\lambda}=\frac{1}{\chi^{2}} \frac{\partial \tilde{\sigma}}{\partial \mu}, \quad \lambda_{\pi}=-\frac{1}{w^{2}}\left(\chi_{\epsilon} D+n \gamma_{\eta}\right), \\
\psi=\frac{n \eta}{w^{3}}, \quad \tilde{\psi}=\frac{2 n \tilde{\eta}}{w^{3}}, \quad \gamma=\frac{\eta\left(n+\chi_{\epsilon}\right)}{\chi w^{2}}, \quad \theta=\frac{1}{\chi w}\left(\frac{\eta n}{w}+\frac{\partial \eta}{\partial \mu}\right), \quad \tilde{\theta}=\frac{1}{\chi w} \frac{\partial \tilde{\eta}}{\partial \mu} .
\end{gathered}
$$

The vertices arising from the first two interactions in eq. (3.13) are the same as given in eq. (2.9). In addition, we get six more vertices
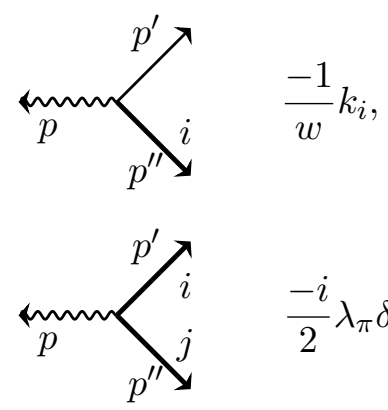

$$
\frac{-i}{2} \lambda_{\pi} \delta_{i j} \boldsymbol{k}^{2}+i \psi k_{i} k_{j}
$$

$-T w \tilde{\psi}\left(\delta_{i j} \boldsymbol{k}^{\prime} \cdot \boldsymbol{k}^{\prime \prime}+k_{i}^{\prime} k_{j}^{\prime \prime}\right)$

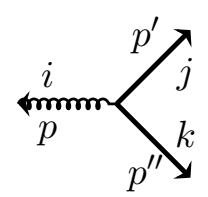
$\frac{-1}{2 w}\left(k_{j} \delta_{i k}+k_{k} \delta_{i j}\right)$

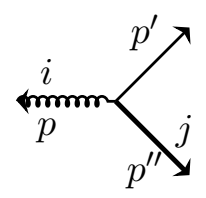

$$
i \gamma \delta_{i j} \boldsymbol{k}^{2}+i \theta\left(\delta_{i j} \boldsymbol{k} \cdot \boldsymbol{k}^{\prime \prime}+k_{j} k_{i}^{\prime \prime}\right)
$$

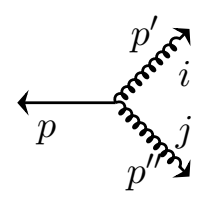

There is another interaction vertex involving the Lagrange multiplier $\Psi_{a}$. However, all the loop diagrams involving this vertex include a purely retarded/advanced loop integral and hence do not contribute to the problem at hand.

Finally, we need the coupling structure to the background fields. Turning on only the $A_{r t}, A_{a t}$ components of the gauge fields, we find

$$
\begin{aligned}
\mathcal{L}_{\text {source }}^{2 \mathrm{pt}}= & \delta n A_{a t}+\chi \partial_{t} \varphi_{a} A_{r t}+\chi A_{r t} A_{a t}, \\
\mathcal{L}_{\text {source }}^{3 \mathrm{pt}}= & \frac{\chi^{\prime}}{\chi} \delta n A_{r t} A_{a t}+\frac{1}{2} \chi^{\prime} \partial_{t} \varphi_{a} A_{r t}^{2}+\frac{1}{2} \chi^{\prime} A_{r t}^{2} A_{a t} \\
& +\left(\frac{\chi^{\prime}}{\chi} \delta n \partial_{t} \varphi_{a}-\frac{1}{\chi} \frac{\partial \sigma}{\partial \mu} \partial^{i} \delta n \partial_{i} \varphi_{a}+i T \frac{\partial \tilde{\sigma}}{\partial \mu} \partial_{i} \varphi_{a} \partial^{i} \varphi_{a}\right) A_{r t}+\left(\frac{\chi}{w} \pi^{i} \partial_{i} \varphi_{a}-X_{a}^{i} \partial_{i} \delta n\right) A_{r t} \\
& +\left(\frac{\chi_{\epsilon}}{w} \pi_{i} \partial_{t} X_{a}^{i}-\frac{2}{w} \frac{\partial \eta}{\partial \mu} \partial^{(i} \pi^{j)} \partial_{i} X_{a j}+2 i T \frac{\partial \tilde{\eta}}{\partial \mu} \partial^{(i} X_{a}^{j)} \partial_{i} X_{a j}\right) A_{r t} .
\end{aligned}
$$


See the appendix A for details of the derivation. Most of these are the same as the ones found in the diffusive case in eq. (2.10), but we do find four additional background interaction vertices, given by

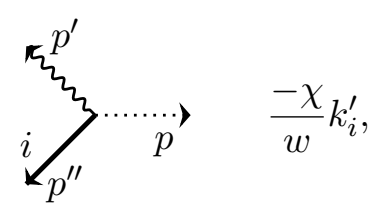

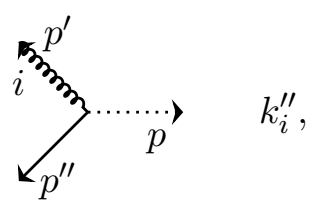

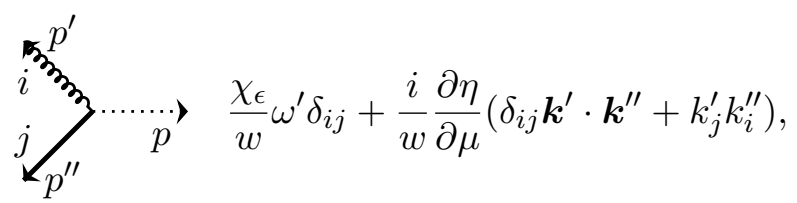

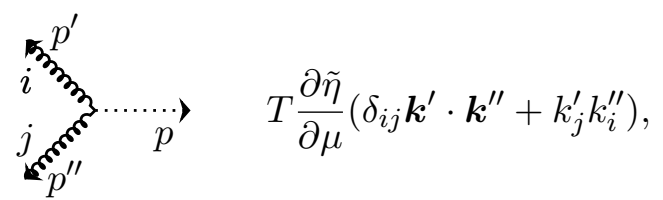

\subsection{One-loop corrections from momentum fluctuations}

We can use this theory to work out the corrections to the density correlation functions arising from momentum fluctuations. The variational formulas in eq. (2.13) are still valid, but with the modified generating functional (see footnote 4 )

$$
\exp W\left[A_{r \mu}, A_{a \mu}\right]=\int \mathcal{D} \delta n \mathcal{D} \varphi_{a} \mathcal{D} \pi^{i} \mathcal{D} X_{a}^{i} \mathcal{D} \Psi_{r} \mathcal{D} \Psi_{a} \exp \left(i \int \mathrm{d}^{d+1} x \mathcal{L}\right) .
$$

This still leads to the same tree-level two-point correlators as reported in eq. (2.6).

\subsubsection{Retarded correlation function}

For the retarded function at one-loop order in diffusive hydrodynamics, we still get contributions from the previous diagrams in eq. (2.14). In addition, we have the following three one-loop diagrams involving momentum fluctuations contributing to the $\left\langle\delta n \varphi_{a}\right\rangle$ propagator:
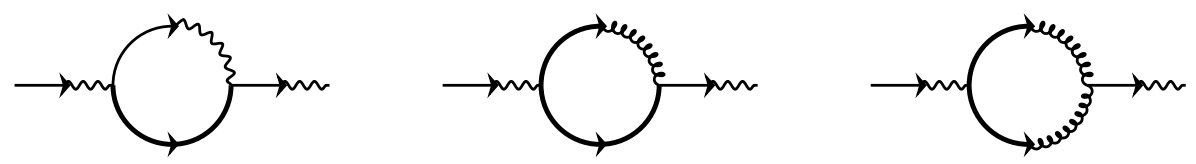

With the incorporation of momentum fluctuations, we also have four new background coupling diagrams:
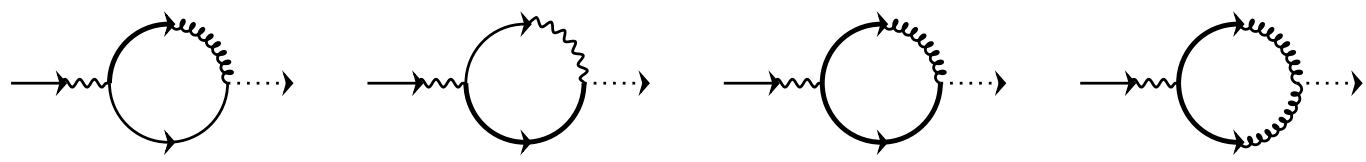

We have ignored the possible diagrams which involve a purely retarded loop and hence do not contribute to the final result. The first diagram in eq. (3.19a) and the first two diagrams in eq. (3.19b) involve leading derivative non-dissipative couplings and dominate in the IR. The remaining four diagrams here and the ones in eq. (2.14) lead to subleading corrections 
in $\boldsymbol{k}^{2}$. The explicit computation of these diagrams can be found in the appendix B. Combining the result from eqs. (2.14) and (3.19), we find that the full one-loop correction to the retarded density correlation function in diffusive hydrodynamics is given by

$$
\begin{aligned}
& G_{n n}^{\mathrm{R}, 1-\operatorname{loop}}(p)=\frac{-T \boldsymbol{k}^{2}}{\left(\omega+i D \boldsymbol{k}^{2}\right)^{2}}\left[\frac{\chi}{w}\left(i \omega \frac{\tilde{\sigma}}{\sigma}+D \boldsymbol{k}^{2}\left(\frac{\tilde{\eta}}{\eta}-\frac{\tilde{\sigma}}{\sigma}\right)\right) \mathcal{I}_{0}(p)\right. \\
& -\lambda\left(\frac{\tilde{\sigma}}{\sigma} i \omega \chi^{2} \lambda-\left(i \omega-D \boldsymbol{k}^{2}\right) \sigma \frac{\partial(\tilde{\sigma} / \sigma)}{\partial \mu}\right) \mathcal{I}_{1}(p) \\
& +i \omega \frac{n_{0} \tilde{\eta}}{w}\left(\lambda_{\pi} \mathcal{J}_{1}(p)+\lambda_{\pi} \mathcal{J}_{2}(p)-\psi \mathcal{J}_{3}(p)-\psi \mathcal{J}_{4}(p)\right) \\
& +D \boldsymbol{k}^{2}\left(\frac{\tilde{\eta}}{w} \chi_{\epsilon}\left(\lambda_{\pi} \mathcal{J}_{1}(p)-\psi \mathcal{J}_{3}(p)\right)+\eta \frac{\partial(\tilde{\eta} / \eta)}{\partial \mu}\left(\lambda_{\pi} \mathcal{J}_{2}(p)-\psi \mathcal{J}_{4}(p)\right)\right) \\
& \left.-\frac{1}{2} \frac{\tilde{\eta}}{w} \chi_{\epsilon}\left(\lambda_{\pi}-\psi\right)\left(i \omega-D \boldsymbol{k}^{2}\right) \mathcal{I}_{3}(0, \boldsymbol{k})\right] \text {. }
\end{aligned}
$$

The integral $\mathcal{I}_{1}(p)$ is defined in eq. (2.16). We define three more similar integrals via

$$
\begin{aligned}
& \mathcal{I}_{0}(p)=\frac{1}{\boldsymbol{k}^{2}} \int^{\Lambda} \frac{\mathrm{d}^{d} \boldsymbol{k}^{\prime}}{(2 \pi)^{d}} \frac{\boldsymbol{k}^{2}-\left(\boldsymbol{k} \cdot \boldsymbol{k}^{\prime}\right)^{2} / \boldsymbol{k}^{\prime 2}}{i \omega-\gamma_{\eta} \boldsymbol{k}^{2}-D\left(\boldsymbol{k}-\boldsymbol{k}^{\prime}\right)^{2}} \\
& \stackrel{d=3}{=} \frac{|\boldsymbol{k}|}{32 \pi D}\left(\frac{2 \sqrt{\gamma_{\eta} D\left(D-\gamma_{\eta}\right)}}{\left(\gamma_{\eta}+D\right)^{2}}+\arccos \left(\frac{\gamma_{\eta}-D}{\gamma_{\eta}+D}\right)\right)+\mathcal{O}(\omega), \\
& \mathcal{I}_{2}(p)=\frac{\boldsymbol{k}^{2}}{2} \int^{\Lambda} \frac{\mathrm{d}^{d} \boldsymbol{k}^{\prime}}{(2 \pi)^{d}} \frac{1}{i \omega-\gamma_{\eta}\left(\boldsymbol{k}^{\prime 2}+\left(\boldsymbol{k}-\boldsymbol{k}^{\prime}\right)^{2}\right)} \stackrel{d=3}{=} \frac{|\boldsymbol{k}|^{3}}{32 \pi D}+\mathcal{O}(\omega), \\
& \mathcal{I}_{3}(p)=\frac{\boldsymbol{k}^{2}}{2} \int^{\Lambda} \frac{\mathrm{d}^{d} \boldsymbol{k}^{\prime}}{(2 \pi)^{d}} \frac{-\boldsymbol{k}^{2} / \boldsymbol{k}^{\prime 2}}{i \omega-\gamma_{\eta}\left(\boldsymbol{k}^{2}+\left(\boldsymbol{k}-\boldsymbol{k}^{\prime}\right)^{2}\right)} \stackrel{d=3}{=} \frac{|\boldsymbol{k}|^{3}}{32 \gamma_{\eta}}+\frac{i \omega|\boldsymbol{k}|}{16 \pi \gamma_{\eta}^{2}}+\mathcal{O}\left(\omega^{2}\right) .
\end{aligned}
$$

For reference, we have computed these integrals explicitly in $d=3$ for small $\omega$, with a hard momentum cutoff $\Lambda$. However, the analyticity results do not depend on the explicit form of the integrals and are valid in arbitrary $d$. In terms of these, the $\mathcal{J}_{1,2,3,4}(p)$ integrals appearing in eq. (3.20) are defined as

$$
\begin{aligned}
& \mathcal{J}_{1}(p)=(d-2) \frac{i \omega}{\gamma_{\eta} \boldsymbol{k}^{2}} \mathcal{I}_{2}(p)-\frac{\left(i \omega-\gamma_{\eta} \boldsymbol{k}^{2}\right)^{2}}{2 \gamma_{\eta}^{2} \boldsymbol{k}^{4}} \mathcal{I}_{3}(p), \\
& \mathcal{J}_{2}(p)=-(d-3) \frac{i \omega-\gamma_{\eta} \boldsymbol{k}^{2}}{\gamma_{\eta} \boldsymbol{k}^{2}} \mathcal{I}_{2}(p)+\frac{\gamma_{\eta} \boldsymbol{k}^{2}}{i \omega}\left(\frac{\left(i \omega-\gamma_{\eta} \boldsymbol{k}^{2}\right)^{3}}{\gamma_{\eta}^{3} \boldsymbol{k}^{6}} \mathcal{I}_{3}(p)+\mathcal{I}_{3}(0, \boldsymbol{k})\right), \\
& \mathcal{J}_{3}(p)=\frac{i \omega\left(i \omega-\gamma_{\eta} \boldsymbol{k}^{2}\right)}{\gamma_{\eta}^{2} \boldsymbol{k}^{4}} \mathcal{I}_{2}(p)+\frac{\left(i \omega-\gamma_{\eta} \boldsymbol{k}^{2}\right)^{3}}{2 \gamma_{\eta}^{3} \boldsymbol{k}^{6}} \mathcal{I}_{3}(p), \\
& \mathcal{J}_{4}(p)=\left(\frac{2 \omega^{2}}{\gamma_{\eta}^{2} \boldsymbol{k}^{4}}-\frac{3 d-1}{d \boldsymbol{k}^{2}}\left(\boldsymbol{k}^{2}-\frac{2 i \omega}{\gamma_{\eta}}\right)\right) \mathcal{I}_{2}(p)-\frac{\gamma_{\eta} \boldsymbol{k}^{2}}{i \omega}\left(\frac{\left(i \omega-\gamma_{\eta} \boldsymbol{k}^{2}\right)^{4}}{\gamma_{\eta}^{4} \boldsymbol{k}^{8}} \mathcal{I}_{3}(p)-\mathcal{I}_{3}(0, \boldsymbol{k})\right) .
\end{aligned}
$$

While $\mathcal{J}_{2}(p)$ and $\mathcal{J}_{4}(p)$ have a $1 / \omega$ appearing in their definitions, they are perfectly regular in the $\omega \rightarrow 0$ limit. 
These expressions are fairly involved. However, if we set $\omega=0$, the one-loop correction to the retarded correlation function reduces to

$$
\begin{aligned}
G_{n n}^{\mathrm{R}, 1-\mathrm{loop}}(0, \mathbf{k})= & \frac{T}{D}\left[\frac{\chi}{w}\left(\frac{\tilde{\eta}}{\eta}-\frac{\tilde{\sigma}}{\sigma}\right) \mathcal{I}_{0}(0, \boldsymbol{k})\right. \\
& \left.\quad-\lambda \sigma \frac{\partial(\tilde{\sigma} / \sigma)}{\partial \mu} \mathcal{I}_{1}(0, \boldsymbol{k})+\eta \frac{\partial(\tilde{\eta} / \eta)}{\partial \mu}\left(\lambda_{\pi} \mathcal{J}_{2}(0, \boldsymbol{k})-\psi \mathcal{J}_{4}(0, \boldsymbol{k})\right)\right], \\
& \stackrel{\mathrm{KMS}}{=} 0 .
\end{aligned}
$$

Hence, we see that the non-analyticities drop out of the retarded correlator in the hydrostatic limit, even after the incorporation of momentum fluctuations, provided the KMS condition is imposed.

\subsubsection{Symmetric correlation function}

We can repeat the same procedure for the symmetric correlation function as well. In addition to the 7 diagrams in eq. (2.18), we find 10 more in the presence of momentum fluctuations:
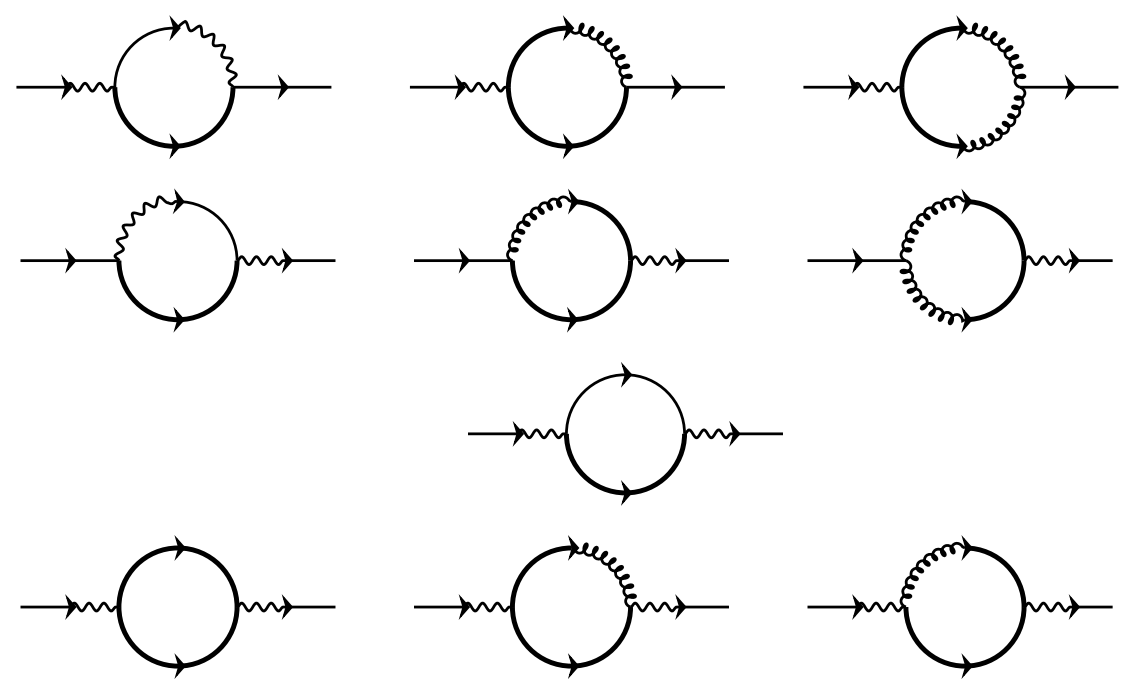

The first diagrams in eqs. (3.24a) to (3.24c) contribute to leading order corrections in $\boldsymbol{k}^{2}$, while the remaining diagrams, along with those in eq. (2.18), contribute at the subleading order. We have computed these diagrams in appendix B, which yield a complicated expression given by

$$
\begin{array}{r}
G_{n n}^{\mathrm{S}, 1-\text { loop }}(\omega, \boldsymbol{k})=-\frac{T^{2} \boldsymbol{k}^{2}}{\left(\omega+i D \boldsymbol{k}^{2}\right)^{2}} \frac{\tilde{\sigma}}{\sigma}\left[\frac{\chi}{w} \frac{\tilde{\eta}}{\eta} \mathcal{I}_{0}(p)-\frac{\tilde{\sigma}}{\sigma} \chi^{2} \lambda^{2} \mathcal{I}_{1}(p)+\frac{D \boldsymbol{k}^{2}}{i \omega} \frac{\tilde{\eta} \chi_{\epsilon}}{w}\left(\lambda_{\pi} \mathcal{J}_{1}(p)-\psi \mathcal{J}_{3}(p)\right)\right. \\
\left.+\frac{n_{0} \tilde{\eta}}{w}\left(\lambda_{\pi} \mathcal{J}_{1}(p)+\lambda_{\pi} \mathcal{J}_{2}(p)-\psi \mathcal{J}_{3}(p)-\psi \mathcal{J}_{4}(p)\right)\right]
\end{array}
$$




$$
\begin{aligned}
& -\frac{T^{2} \tilde{\eta} / w \boldsymbol{k}^{2}}{\omega^{2}+D^{2} \boldsymbol{k}^{4}}\left(\frac{\tilde{\eta}}{\eta}-\frac{\tilde{\sigma}}{\sigma}\right)\left[\chi_{\epsilon} \frac{D \boldsymbol{k}^{2}}{i \omega}\left(\lambda_{\pi} \mathcal{J}_{1}(p)-\psi \mathcal{J}_{3}(p)\right)\right. \\
& \left.+n_{0}\left(\lambda_{\pi} \mathcal{J}_{1}(p)+\lambda_{\pi} \mathcal{J}_{2}(p)-\psi \mathcal{J}_{3}(p)-\psi \mathcal{J}_{4}(p)\right)\right] \\
& -\frac{2 i T^{2} \eta \tilde{\sigma} / \chi \boldsymbol{k}^{4}}{\left(\omega+i D \boldsymbol{k}^{2}\right)\left(\omega^{2}+D^{2} \boldsymbol{k}^{4}\right)} \frac{\partial(\tilde{\eta} / \eta)}{\partial \mu}\left(\lambda_{\pi} \mathcal{J}_{2}(p)-\psi \mathcal{J}_{4}(p)\right) \\
& + \text { complex conjugate. }
\end{aligned}
$$

When the KMS symmetry is respected, the result simplifies and the remaining contributions match the relevant terms in the retarded correlation function, in accordance with the fluctuation-dissipation theorem (1.3). We have computed these terms in the $\omega=0$ limit and verified that the answer is well-defined and still non-analytic, even in the presence of KMS. For $d=3$, the results have been explicitly presented in the introduction.

\section{Discussion}

In this paper, we explored the spatial analyticity of hydrodynamic correlation functions at loop-level, using the Schwinger-Keldysh EFT formalism that incorporates nonlinear stochastic fluctuations consistent with the KMS condition. We computed the complete one-loop correction to the retarded and symmetric two-point functions of charge density, due to diffusive density and transverse momentum fluctuations. The symmetric correlation function is found to be generically non-analytic, owing to the absence of a characteristic mass scale in the EFT. Despite this, we find that the retarded correlation function nontrivially respects the spatial analyticity requirement expected in the hydrostatic limit due to thermal screening. Notably, the analyticity behaviour crucially depends on the dynamical KMS symmetry in the EFT, that leads to a series of non-trivial cancellations of non-analytic terms in the hydrostatic limit. Our final results are summarized in section 1.1.

We performed the majority of loop calculations in this paper in generic spatial dimensions and away from the hydrostatic limit $(\omega \neq 0)$. This analysis can be recycled to study the phenomenon of long-time tails at finite- $\omega$ finite- $\boldsymbol{k}$ in relativistic hydrodynamics in the presence of momentum fluctuations, closely following a similar analysis for scalar diffusion model in [10]. These results will appear in a companion paper [19].

During the course of our analysis, we developed a novel EFT model for "incompressible" diffusive hydrodynamics that is consistent with the KMS condition beyond the linearised fluctuations, accounting for the presence of arbitrary non-linear interactions. This model can be understood as systematically integrating out the "higher-energy" sound modes from the hydrodynamic setup and allows us to study the effects of momentum fluctuations in a controlled setting, which is expected to have far-reaching applications beyond the context of the present work.

Although not the focus of this paper, in computing the symmetric correlation functions, we have exhibited specific non-analyticities that are computable, independent of the UV cutoff, and determined by thermodynamic parameters and hydrodynamic transport coefficients. As such, symmetric correlators are interesting quantities in themselves. 
One approach to computing transport coefficients within the microscopic theory utilizes Euclidean correlators on a finite size lattice. Although the physical content of Euclidean correlators most directly maps to Minkowskian retarded correlators, considering the static limit of the symmetric correlator may be helpful for extracting transport coefficients via finite size scaling.

In terms of future directions, its important to note that we have limited our considerations in this paper to generic thermal systems that are assumed to have a finite screened spatial correlation length. There are of course physical systems in which long-range correlations survive and may lead to physical non-analyticities in retarded correlators. Examples include conformal fixed points, and superfluids, which are known to require additional degrees of freedom within the hydrostatic generating functional. It would be interesting to explore how spatial non-analyticities re-emerge in such theories. The analysis can also be extended in the context of non-relativistic hydrodynamics using recently developed field theory methods [7].

Finally, we have argued that the KMS condition, associated with the existence and properties of the thermal equilibrium state, is the critical ingredient in ensuring the spatial analyticity of the retarded correlators in the hydrostatic limit. While this is, of course, consistent with the assumptions underlying the derivative expansion for the hydrostatic generating functional, it is natural to ask whether it can be imposed in a more direct and local manner within the EFT itself. Currently, the KMS condition is formulated as a non-local discrete symmetry condition to be imposed on the Schwinger-Keldysh EFT, and it would be interesting to understand if a more covariant formalism would allow for it to be imposed directly in writing down the EFT Lagrangian. In particular, we have only showed the analyticity of retarded correlators at one-loop level. It will be interesting to explore whether such a result can be derived at the full non-perturbative level in the EFT formalism of hydrodynamics.

\section{Acknowledgments}

AS would like to acknowledge the hospitality of the Centre for Particle Theory at Durham University, and the Department of Physics at McGill University, where parts of this work were carried out. This work is supported in part by NSERC, Canada. Research at Perimeter Institute is supported in part by the Government of Canada through the Department of Innovation, Science and Economic Development Canada and by the Province of Ontario through the Ministry of Colleges and Universities.

\section{A The effective action for diffusive hydrodynamics}

In this appendix, we provide further details on the computation of the perturbative effective action for relativistic diffusive hydrodynamics presented in section 3.2, starting from the Schwinger-Keldysh effective field theory.

Let us start with the effective action for full hydrodynamics in eq. (3.4), with the constraints eq. (3.7). We need to expand the effective action order-by-order in the dynamical 
fields $\delta \beta^{\mu}, \delta \Lambda_{\beta}, X_{a}^{\mu}, \varphi_{a}$, and the background fields $A_{r t}, A_{a t}$. Segregating space and time derivatives in the effective Lagrangian eq. (3.4), we can express it as

$$
\begin{aligned}
\mathcal{L}= & \left(n_{0}+\delta \hat{n}\right) A_{a t}-\varphi_{a} \partial_{t} \delta \hat{n}-X_{a}^{i} \partial_{t} \hat{\pi}_{i}+J^{i} \partial_{i} \varphi_{a}+T^{i j} \partial_{i} X_{a j}+\delta \hat{n} X_{a}^{i} \partial_{i} A_{r t} \\
& +i T \tilde{\sigma} \partial_{i} \varphi_{a} \partial^{i} \varphi_{a}+i T \tilde{\eta}\left(\partial^{i} X_{a}^{j} \partial_{i} X_{a j}+\partial^{j} X_{a}^{i} \partial_{i} X_{a j}\right)+\Psi_{a} \partial_{i} \frac{u^{i}}{T}+\Psi_{r} \partial_{i} X_{a}^{i}+\ldots
\end{aligned}
$$

where we have only kept terms up to cubic order in dynamical and background fields, and defined

$$
\begin{aligned}
\delta \hat{n} & \equiv J^{t}-n_{0}+2 i T \tilde{\sigma} u^{i} \partial_{i} \varphi_{a} \\
& =n-n_{0}+\frac{1}{2} n_{0} \boldsymbol{u}^{2}-\sigma_{0} u^{i}\left(\partial_{i} \mu-\partial_{i} A_{r t}\right)+2 i T_{0} \tilde{\sigma}_{0} u^{i} \partial_{i} \varphi_{a}+\cdots, \\
\hat{\pi}^{i} & \equiv T^{t i}+2 i T \tilde{\eta}\left(u^{k} \partial_{k} X_{a}^{i}+u_{k} \partial^{i} X_{a}^{k}\right) \\
& =w u^{i}-\eta_{0}\left(u^{k} \partial_{k} u^{i}+u_{k} \partial^{i} u^{k}\right)+2 i T_{0} \tilde{\eta}_{0}\left(u^{k} \partial_{k} X_{a}^{i}+u_{k} \partial^{i} X_{a}^{k}\right)+\cdots
\end{aligned}
$$

Here $w=\epsilon+p$ is the enthalpy density. The subscript " 0 " denotes that the corresponding quantity is evaluated at equilibrium. These are the true non-classical conserved charge and momentum densities. We can further split these into

$$
\begin{aligned}
& \delta \hat{n}=\delta n+\chi A_{r t}+\frac{\chi^{\prime}}{\chi} \delta n A_{r t}+\frac{1}{2} \chi^{\prime} A_{r t}^{2}+\cdots, \\
& \hat{\pi}^{i}=\pi^{i}+\frac{\chi_{\epsilon}}{w_{0}} \pi^{i} A_{r t}+\cdots,
\end{aligned}
$$

with $\delta n$ and $\pi^{i}$ being the respective versions in the absence of background fields,

$$
\begin{aligned}
\delta n & =\chi \delta \mu+\frac{1}{2} \chi^{\prime} \delta \mu^{2}+\frac{1}{2} \chi_{\epsilon} \boldsymbol{u}^{2}-\sigma_{0} u^{i} \partial_{i} \delta \mu+2 i T_{0} \tilde{\sigma}_{0} u^{i} \partial_{i} \varphi_{a}+\cdots, \\
\pi^{i} & =w_{0} u^{i}+\chi_{\epsilon} u^{i} \delta \mu-\eta_{0}\left(\frac{1}{2} \partial^{i} \boldsymbol{u}^{2}+u^{j} \partial_{j} u^{i}\right)+2 i T_{0} \tilde{\eta}_{0}\left(u_{j} \partial^{i} X_{a}^{j}+u^{j} \partial_{j} X_{a}^{i}\right)+\cdots,
\end{aligned}
$$

where $\delta \mu=T_{0} \delta \Lambda_{\beta}$ and $u^{i}=T_{0} \delta \beta^{i}$, and we have used

$$
\chi=\left.\frac{\partial n}{\partial \mu}\right|_{0}, \quad \chi_{\epsilon}=n_{0}+\left.\frac{\partial \epsilon}{\partial \mu}\right|_{0}=n_{0}+\left.\mu \frac{\partial n}{\partial \mu}\right|_{0}+\left.T \frac{\partial n}{\partial T}\right|_{0}, \quad \chi^{\prime}=\left.\frac{\partial^{2} n}{\partial \mu^{2}}\right|_{0} .
$$

Note that $\mu=\mu_{0}+\delta \mu+A_{r t}+\mu_{0} / 2 \boldsymbol{u}^{2}$. In the following, we shall find it easier to work with $\delta n, \pi^{i}$ instead of $\delta \mu, u^{i}$, because this eliminates time-derivative terms from the interaction Lagrangian. We can find the inverted relations

$$
\begin{aligned}
\delta \mu & =\frac{1}{\chi} \delta n-\frac{\chi^{\prime}}{2 \chi^{3}} \delta n^{2}-\frac{\chi_{\epsilon}}{2 \chi w_{0}^{2}} \pi^{2}+\frac{\sigma_{0}}{\chi^{2} w_{0}} \pi^{i} \partial_{i} \delta n-\frac{2 i T_{0} \tilde{\sigma}_{0}}{\chi w_{0}} \pi^{i} \partial_{i} \varphi_{a}+\cdots \\
u^{i} & =\frac{1}{w_{0}} \pi^{i}-\frac{\chi_{\epsilon}}{\chi w_{0}^{2}} \pi^{i} \delta n+\frac{\eta_{0}}{w_{0}^{3}}\left(\frac{1}{2} \partial^{i} \pi^{2}+\pi^{j} \partial_{j} \pi^{i}\right)-\frac{2 i T_{0} \tilde{\eta}_{0}}{w_{0}^{2}}\left(\pi_{j} \partial^{i} X_{a}^{j}+\pi^{j} \partial_{j} X_{a}^{i}\right)+\cdots
\end{aligned}
$$

The divergenceless condition in eq. (3.7) implies that

$$
\partial_{i} \pi^{i}=\frac{\chi_{\epsilon}}{\chi w_{0}} \pi^{i} \partial_{i} \delta n+\ldots,
$$

where we have dropped the higher-derivative terms. 
Using these expressions, we can go back and express $J^{i}$ and $T^{i j}$ also in terms of the fluctuations $\delta n$ and $\pi^{i}$, and the background fields $A_{r t}$ and $A_{a t}$. We obtain

$$
\begin{aligned}
J^{i}= & n(T, \mu) u^{i}-\sigma(T, \mu) \delta^{i j}\left(T \partial_{j}\left(\frac{\mu}{T}\right)-\partial_{j} A_{r t}\right)-T_{0} \sigma_{0} u^{i} \partial_{t}\left(\frac{\mu}{T}\right)+\ldots \\
= & \frac{n_{0}}{w_{0}} \pi^{i}-\frac{\sigma_{0}}{\chi} \partial^{i} \delta n-\left(\frac{n_{0} \chi_{\epsilon}}{\chi w_{0}^{2}}-\frac{1}{w_{0}}\right) \pi^{i} \delta n+\frac{n_{0} \eta_{0}}{w_{0}^{3}}\left(\frac{1}{2} \partial^{i} \pi^{2}+\pi^{j} \partial_{j} \pi^{i}\right) \\
& -\left.\frac{1}{2 \chi} \frac{\partial(\sigma / \chi)}{\partial \mu}\right|_{0} \partial^{i} \delta n^{2}+\frac{\sigma_{0} \chi_{\epsilon}}{2 \chi w_{0}^{2}} \partial^{i} \pi^{2}-\frac{2 i T_{0} \tilde{\eta}_{0} n_{0}}{w_{0}^{2}}\left(\pi_{j} \partial^{i} X_{a}^{j}+\pi^{j} \partial_{j} X_{a}^{i}\right) \\
& +\frac{\chi}{w_{0}} A_{r t} \pi^{i}-\left.\frac{1}{\chi} \frac{\partial \sigma}{\partial \mu}\right|_{0} A_{r t} \partial^{i} \delta n-\left\{\frac{\sigma_{0}}{\chi w_{0}} \pi^{i} \partial_{\mu} J^{\mu}\right\}+\ldots,
\end{aligned}
$$

and

$$
\begin{aligned}
T^{i j}= & w_{0} u^{i} u^{j}+p(T, \mu) \delta^{i j}-\eta(T, \mu)\left(\partial^{i} u^{j}+\partial^{j} u^{i}\right)-\eta_{0}\left(u^{i} \partial_{t} u^{j}+u^{j} \partial_{t} u^{i}\right)+\ldots \\
= & -\frac{2 \eta_{0}}{w_{0}} \partial^{(i} \pi^{j)}+\frac{1}{w_{0}} \pi^{i} \pi^{j}-\left.\frac{2}{\chi w_{0}} \frac{\partial \eta}{\partial \mu}\right|_{0} \delta n \partial^{(i} \pi^{j)}+\frac{2 \eta_{0} \chi_{\epsilon}}{\chi w_{0}^{2}} \delta n \partial^{(i} \pi^{j)}+\left(\frac{2 \eta_{0}\left(n_{0}+\chi_{\epsilon}\right)}{\chi w_{0}^{2}}\right) \pi^{(i} \partial^{j)} \delta n \\
& -\left.\frac{2}{w_{0}} \frac{\partial \eta}{\partial \mu}\right|_{0} A_{r t} \partial^{(i} \pi^{j)}-\left\{\frac{2 \eta_{0}}{w_{0}^{2}} \pi^{(i}\left(\partial_{\mu} T^{j) \mu}-F_{r}^{j) \lambda} J_{\lambda}\right)\right\}+(\ldots) \delta^{i j}+\ldots
\end{aligned}
$$

We have not expanded the trace part of $T^{i j}$ as it couples to $\partial_{i} X_{a}^{i}$ in the effective action eq. (3.4) and does not contribute in the diffusive limit eq. (3.7). We have expressed the terms involving time-derivatives using the equations of motion in the expressions above (denoted in braces), which can be removed by redefining $\varphi_{a}$ and $X_{a}^{i}$ as

$$
\varphi_{a} \rightarrow \varphi_{a}-\frac{\sigma_{0}}{\chi w_{0}} \pi^{i} \partial_{i} \varphi_{a}, \quad X_{a}^{i} \rightarrow X_{a}^{i}-\frac{\eta_{0}}{w_{0}^{2}}\left(\pi_{k} \partial^{i} X_{a}^{k}+\pi^{k} \partial_{k} X_{a}^{i}\right) .
$$

Note that the imaginary part in $J^{i}$ is not true statistical flux (which will be obtained by varying the action), but merely one of the contributions to the effective action.

The free, interaction, and background coupling part of the Lagrangian in section 3.2, eqs. (3.11), (3.13), and (3.16), follow directly from here by substituting for $T^{i j}, J^{i}, \delta \hat{n}$, and $\hat{\pi}^{i}$ into eq. (A.1).

\section{B Loop calculations}

In this appendix, we provide explicit details of the calculations involved in computing the loop integrals presented in the main text.

One-loop corrections to the retarded and symmetric correlation functions of charge density can be computed using the variational formulae in eq. (2.13). Starting with the retarded function, we first need to compute the one-loop diagrams correcting the $\left\langle\delta n \varphi_{a}\right\rangle$ propagator given in eqs. (2.14a) and (3.19a). Let us denote the amputated version, i.e. diagrams ignoring the external legs, by $i \Gamma_{a r}(p)$. Next, we have diagrams involving a nonlinear background field coupling from eqs. (2.14b) and (3.19b), whose amputated version is denoted by $i \Gamma_{a r}^{\prime}(p)$. The full one-loop retarded correlation function is given in terms of these as

$$
G_{n n}^{\mathrm{R}, 1-\operatorname{loop}}(p)=\frac{i \omega \chi \Gamma_{a r}(p)}{\left(i \omega-D \boldsymbol{k}^{2}\right)^{2}}-\frac{\Gamma_{a r}^{\prime}(p)}{i \omega-D \boldsymbol{k}^{2}} .
$$


For completeness, we note that the one-loop correction to the advanced correlation function will be given by its complex conjugate,

$$
G_{n n}^{\mathrm{A}, 1-\operatorname{loop}}(p)=\frac{-i \omega \chi \Gamma_{a r}^{*}(p)}{\left(i \omega+D \boldsymbol{k}^{2}\right)^{2}}+\frac{\Gamma_{a r}^{* *}(p)}{i \omega+D \boldsymbol{k}^{2}} .
$$

On the other hand, for the symmetric function, we need to compute the diagrams in eqs. (2.18) and (3.24). Note that the diagrams in eqs. (2.18a) and (3.24a) are the same as those for the retarded function in eqs. (2.14a) and (3.19a), except with different external legs. Hence, their amputated version is still given by $i \Gamma_{a r}(p)$. Furthermore, the diagrams in eqs. (2.18b) and (3.24b) are merely their complex conjugates, and hence their amputated version is given by $-i \Gamma_{a r}^{*}(p)$. Therefore, the only diagrams that we need to compute for the symmetric function are given in eqs. $(2.18 \mathrm{c}),(3.24 \mathrm{c})$ and $(3.24 \mathrm{~d})$. We denote their amputated version by $\Gamma_{a a}$. In total, the one-loop correction to the symmetric function is given by

$$
G_{n n}^{\mathrm{S}, 1-\operatorname{loop}}(p)=\frac{-\Gamma_{a a}(p)}{\omega^{2}+D^{2} \boldsymbol{k}^{4}}-\frac{2 T \tilde{\sigma} \boldsymbol{k}^{2} \Gamma_{a r}(p)}{\left(i \omega-D \boldsymbol{k}^{2}\right)\left(\omega^{2}+D^{2} \boldsymbol{k}^{4}\right)}+\frac{2 T \tilde{\sigma} \boldsymbol{k}^{2} \Gamma_{a r}^{*}(p)}{\left(i \omega+D \boldsymbol{k}^{2}\right)\left(\omega^{2}+D^{2} \boldsymbol{k}^{4}\right)} .
$$

In the remainder of this appendix, we explicitly compute $\Gamma_{a r}(p), \Gamma_{a r}^{\prime}(p)$, and $\Gamma_{a a}(p)$.

For clarity, we use the following notation: $F(p)=\omega+i D \boldsymbol{k}^{2}, G(p)=\omega+i \gamma_{\eta} \boldsymbol{k}^{2}$, $\bar{k}^{\prime i j}=\delta^{i j}-k^{\prime i} k^{\prime j} / \boldsymbol{k}^{\prime 2}, \bar{k}^{\prime \prime i j}=\delta^{i j}-k^{\prime \prime i} k^{\prime \prime j} / \boldsymbol{k}^{\prime \prime 2}$, along with $\omega^{\prime \prime}=\omega-\omega^{\prime}$ and $k^{\prime \prime i}=k^{i}-k^{i}$. We also denote the frequency-momentum integrals compactly as $\int_{p^{\prime}}=\int \mathrm{d} \omega^{\prime} \mathrm{d}^{d} \boldsymbol{k}^{\prime} /(2 \pi)^{d+1}$, and just the momentum integrals as $\int_{k^{\prime}}=\int \mathrm{d}^{d} \boldsymbol{k}^{\prime} /(2 \pi)^{d}$. Momentum integrals are performed with a hard UV-cutoff at $\left|\boldsymbol{k}^{\prime}\right|=\Lambda$ and all the cutoff-dependent terms are ignored. We make extensive use of the change of variables $\omega^{\prime} \rightarrow \omega^{\prime \prime}, \boldsymbol{k}^{\prime} \rightarrow \boldsymbol{k}^{\prime \prime}$ to simplify the integrals, which leads to identities such as

$$
\begin{aligned}
\int_{p^{\prime}} \frac{\boldsymbol{k}^{\prime 2}}{F\left(p^{\prime}\right) F\left(p^{\prime \prime}\right)}=\frac{1}{2} \int_{k^{\prime}} \frac{\boldsymbol{k}^{\prime 2}+\boldsymbol{k}^{\prime \prime 2}}{i \omega-D\left(\boldsymbol{k}^{\prime 2}+\boldsymbol{k}^{\prime 2}\right)}=\frac{i \omega}{2 D} \int_{p^{\prime}} \frac{1}{F\left(p^{\prime}\right) F\left(p^{\prime \prime}\right)}, \\
\int_{p^{\prime}} \frac{\boldsymbol{k}^{\prime} \cdot \boldsymbol{k}^{\prime \prime}}{F\left(p^{\prime}\right) F\left(p^{\prime \prime}\right)}=\frac{1}{2} \int_{k^{\prime}} \frac{\boldsymbol{k}^{2}-\left(\boldsymbol{k}^{\prime 2}+\boldsymbol{k}^{\prime \prime 2}\right)}{i \omega-D\left(\boldsymbol{k}^{\prime 2}+\boldsymbol{k}^{\prime 2}\right)}=\frac{-i F(p)}{2 D} \int_{p^{\prime}} \frac{1}{F\left(p^{\prime}\right) F\left(p^{\prime \prime}\right)},
\end{aligned}
$$

up to cutoff-dependent terms.

\section{B.1 Computation of $\boldsymbol{\Gamma}_{a r}$}

Let us start with the contributions from the two density fluctuation diagrams in eq. (2.14a). This is a generalization of the calculation in [9] to account for $\tilde{\sigma} \neq \sigma$. We find

$$
\begin{aligned}
i \Gamma_{a r}(p) & \sim-2 T \tilde{\sigma} \lambda^{2} \boldsymbol{k}^{2} \int_{p^{\prime}} \frac{\boldsymbol{k}^{\prime 2} \boldsymbol{k}^{\prime \prime 2}}{F\left(p^{\prime}\right)\left|F\left(p^{\prime \prime}\right)\right|^{2}}-i T \chi \lambda \tilde{\lambda} \boldsymbol{k}^{2} \int_{p^{\prime}} \frac{\boldsymbol{k}^{\prime} \cdot \boldsymbol{k}^{\prime \prime}}{F\left(p^{\prime}\right) F\left(p^{\prime \prime}\right)} \\
& =-i T \chi \lambda \boldsymbol{k}^{2} \int_{p^{\prime}} \frac{\lambda \tilde{\sigma} / \sigma \boldsymbol{k}^{\prime 2}+\tilde{\lambda} \boldsymbol{k}^{\prime} \cdot \boldsymbol{k}^{\prime \prime}}{F\left(p^{\prime}\right) F\left(p^{\prime \prime}\right)} \\
& =-\frac{i T \chi \lambda}{D}\left(i \omega \lambda \frac{\tilde{\sigma}}{\sigma}-i F(p) \tilde{\lambda}\right) \mathcal{I}_{1}(p) .
\end{aligned}
$$


In the second step, we have split the tree " $r r$ " propagator into a sum of "ra" and "ar" propagators according to eq. (2.6), and noted that the "ar" piece does not contribute due to a purely retarded loop. The integral $\mathcal{I}_{1}(p)$ is defined in eq. (2.16). Next, we have contributions coming from the single leading-order "mixed" fluctuation diagram in eq. (3.19a)

$$
i \Gamma_{a r}(p) \sim \frac{2 T \tilde{\eta}}{w^{2}} \int_{p^{\prime}} \frac{\boldsymbol{k}^{\prime 2} \bar{k}^{\prime i j} k_{i} k_{j}^{\prime \prime}}{\left|G\left(p^{\prime}\right)\right|^{2} F\left(p^{\prime \prime}\right)}=\frac{i T}{w} \frac{\tilde{\eta}}{\eta} \int_{p^{\prime}} \frac{\boldsymbol{k}^{2}-\left(\boldsymbol{k} \cdot \boldsymbol{k}^{\prime}\right)^{2} / \boldsymbol{k}^{\prime 2}}{G\left(p^{\prime}\right) F\left(p^{\prime \prime}\right)}=\frac{i T \boldsymbol{k}^{2}}{w} \frac{\tilde{\eta}}{\eta} \mathcal{I}_{0}(p)
$$

The integral $\mathcal{I}_{0}(p)$ is defined in eq. (3.21). Finally, we have two momentum-fluctuation diagrams in eq. (3.19a) leading to

$$
\begin{aligned}
i \Gamma_{a r}(p) \sim & 2 T \tilde{\eta} \int_{p^{\prime}} \frac{\boldsymbol{k}^{\prime \prime 2}\left(\lambda_{\pi} \boldsymbol{k}^{2} \delta_{i j}-2 \psi k_{i} k_{j}\right) \bar{k}^{\prime i k} \bar{k}^{\prime \prime \prime l}\left(\gamma \delta_{k l} \boldsymbol{k}^{\prime 2}+\theta\left(\delta_{k l} \boldsymbol{k}^{\prime} \cdot \boldsymbol{k}^{\prime \prime}+k_{l}^{\prime} k_{k}^{\prime \prime}\right)\right)}{G\left(p^{\prime}\right)\left|G\left(p^{\prime \prime}\right)\right|^{2}} \\
& -i T w \tilde{\theta} \int_{p^{\prime}} \frac{\left(\lambda_{\pi} \boldsymbol{k}^{2} \delta_{i j}-2 \psi k_{i} k_{j}\right) \bar{k}^{\prime i k} \bar{k}^{\prime \prime j l}\left(\delta_{k l} \boldsymbol{k}^{\prime} \cdot \boldsymbol{k}^{\prime \prime}+k_{l}^{\prime} k_{k}^{\prime \prime}\right)}{G\left(p^{\prime}\right) G\left(p^{\prime \prime}\right)} \\
= & i T w \int_{p^{\prime}} \frac{\left(\lambda_{\pi} \boldsymbol{k}^{2} \delta_{i j}-2 \psi k_{i} k_{j}\right) \bar{k}^{\prime i k} \bar{k}^{\prime \prime j l}\left(\frac{\tilde{\eta}}{\eta} \gamma \delta_{k l} \boldsymbol{k}^{\prime 2}+\left(\frac{\tilde{\eta}}{\eta} \theta-\tilde{\theta}\right)\left(\delta_{k l} \boldsymbol{k}^{\prime} \cdot \boldsymbol{k}^{\prime \prime}+k_{k} k_{l}\right)\right)}{G\left(p^{\prime}\right) G\left(p^{\prime \prime}\right)} \\
= & i T w \boldsymbol{k}^{2}\left(\frac{\tilde{\eta}}{\eta} \lambda_{\pi} \gamma \mathcal{J}_{1}(p)+\lambda_{\pi}\left(\frac{\tilde{\eta}}{\eta} \theta-\tilde{\theta}\right) \mathcal{J}_{2}(p)-\frac{\tilde{\eta}}{\eta} \psi \gamma \mathcal{J}_{3}(p)-\psi\left(\frac{\tilde{\eta}}{\eta} \theta-\tilde{\theta}\right) \mathcal{J}_{4}(p)\right) .
\end{aligned}
$$

The integrals $\mathcal{J}_{1,2,3,4}(p)$ are defined as

$$
\begin{aligned}
\mathcal{J}_{1}(p) & =\int_{p^{\prime}} \frac{\boldsymbol{k}^{\prime \prime 2} \bar{k}^{\prime i k} \bar{k}^{\prime \prime j l} \delta_{i j} \delta_{k l}}{G\left(p^{\prime}\right) G\left(p^{\prime \prime}\right)}=\int_{p^{\prime}} \frac{\boldsymbol{k}^{\prime \prime 2}(d-2)+\left(\boldsymbol{k}^{\prime} \cdot \boldsymbol{k}^{\prime \prime}\right)^{2} / \boldsymbol{k}^{\prime 2}}{G\left(p^{\prime}\right) G\left(p^{\prime \prime}\right)} \\
\mathcal{J}_{2}(p) & =\int_{p^{\prime}} \frac{\bar{k}^{\prime i k} \bar{k}^{\prime \prime j l} \delta_{i j}\left(\boldsymbol{k}^{\prime} \cdot \boldsymbol{k}^{\prime \prime} \delta_{k l}+k_{k} k_{l}\right)}{G\left(p^{\prime}\right) G\left(p^{\prime \prime}\right)}=\int_{p^{\prime}} \frac{\left(\boldsymbol{k}^{\prime} \cdot \boldsymbol{k}^{\prime \prime}\right)\left((d-3)+2\left(\boldsymbol{k}^{\prime} \cdot \boldsymbol{k}^{\prime \prime}\right)^{2} /\left(\boldsymbol{k}^{\prime 2} \boldsymbol{k}^{\prime \prime 2}\right)\right)}{G\left(p^{\prime}\right) G\left(p^{\prime \prime}\right)}, \\
\mathcal{J}_{3}(p) & =\frac{2}{\boldsymbol{k}^{2}} \int_{p^{\prime}} \frac{\boldsymbol{k}^{\prime \prime 2} \bar{k}^{\prime i k} \bar{k}^{\prime \prime j l} \delta_{i j} k_{k} k_{l}}{G\left(p^{\prime}\right) G\left(p^{\prime \prime}\right)}=\frac{2}{\boldsymbol{k}^{2}} \int_{p^{\prime}} \frac{\left(\boldsymbol{k}^{\prime} \cdot \boldsymbol{k}^{\prime \prime}\right)\left(-\boldsymbol{k}^{\prime \prime 2}+\left(\boldsymbol{k}^{\prime} \cdot \boldsymbol{k}^{\prime \prime}\right)^{2} / \boldsymbol{k}^{\prime 2}\right)}{G\left(p^{\prime}\right) G\left(p^{\prime \prime}\right)} \\
\mathcal{J}_{4}(p) & =\frac{2}{\boldsymbol{k}^{2}} \int_{p^{\prime}} \frac{\bar{k}^{\prime i k} \bar{k}^{\prime \prime j l} k_{i} k_{j}\left(\boldsymbol{k}^{\prime} \cdot \boldsymbol{k}^{\prime \prime} \delta_{k l}+k_{k} k_{l}\right)}{G\left(p^{\prime}\right) G\left(p^{\prime \prime}\right)} \\
& =\frac{2}{\boldsymbol{k}^{2}} \int_{p^{\prime}} \frac{\boldsymbol{k}^{\prime 2} \boldsymbol{k}^{\prime \prime 2}-3\left(\boldsymbol{k}^{\prime} \cdot \boldsymbol{k}^{\prime \prime}\right)^{2}+2\left(\boldsymbol{k}^{\prime} \cdot \boldsymbol{k}^{\prime \prime}\right)^{4} /\left(\boldsymbol{k}^{\prime 2} \boldsymbol{k}^{\prime \prime 2}\right)}{G\left(p^{\prime}\right) G\left(p^{\prime \prime}\right)}
\end{aligned}
$$

Employing identities similar to those in eq. (B.4), these four integrals can be related to just two integrals $\mathcal{I}_{2}(p), \mathcal{I}_{3}(p)$, as given in eq. (3.22). 


\section{B.2 Computation of $\Gamma_{a r}^{\prime}$}

For the two density fluctuation background coupling diagrams in eq. (2.14b), we find

$$
\begin{aligned}
i \Gamma_{a r}^{\prime}(p) & \sim \frac{2 T \tilde{\sigma} \lambda}{\chi} \boldsymbol{k}^{2} \int_{p^{\prime}} \frac{\boldsymbol{k}^{\prime \prime 2}\left(\sigma^{\prime} \boldsymbol{k}^{\prime} \cdot \boldsymbol{k}^{\prime \prime}+\chi^{\prime} i \omega^{\prime}\right)}{F\left(p^{\prime}\right)\left|F\left(p^{\prime \prime}\right)\right|^{2}}-i T \chi^{2} \lambda \tilde{\lambda} \boldsymbol{k}^{2} \int_{p^{\prime}} \frac{\boldsymbol{k}^{\prime} \cdot \boldsymbol{k}^{\prime \prime}}{F\left(p^{\prime}\right) F\left(p^{\prime \prime}\right)} \\
& =i T \lambda \boldsymbol{k}^{2} \int_{p^{\prime}} \frac{\frac{\tilde{\sigma}}{\sigma} \chi^{\prime} i \omega^{\prime}+\left(\frac{\tilde{\sigma}}{\sigma} \frac{\partial \sigma}{\partial \mu}-\frac{\partial \tilde{\sigma}}{\partial \mu}\right) \boldsymbol{k}^{\prime} \cdot \boldsymbol{k}^{\prime \prime}}{F\left(p^{\prime}\right) F\left(p^{\prime \prime}\right)} \\
& =\frac{i T \lambda}{D}\left(\frac{\tilde{\sigma}}{\sigma} \chi^{\prime} D i \omega+i F(p) \sigma \frac{\partial(\tilde{\sigma} / \sigma)}{\partial \mu}\right) \mathcal{I}_{1}(p) .
\end{aligned}
$$

The two leading-order mixed fluctuation diagrams in eq. (3.19b) lead to

$$
\begin{aligned}
i \Gamma_{a r}^{\prime}(p) & \sim \frac{2 T \tilde{\eta} \chi}{w^{2}} \int_{p^{\prime}} \frac{\boldsymbol{k}^{\prime 2} \bar{k}^{\prime i j} k_{i}^{\prime \prime} k_{j}^{\prime \prime}}{\left|G\left(p^{\prime}\right)\right|^{2} F\left(p^{\prime \prime}\right)}-\frac{2 T \tilde{\sigma}}{w} \int_{p^{\prime}} \frac{\boldsymbol{k}^{\prime \prime 2} \bar{k}^{\prime i j} k_{i} k_{j}^{\prime \prime}}{G\left(p^{\prime}\right)\left|F\left(p^{\prime \prime}\right)\right|^{2}} \\
& =\frac{i T \chi}{w}\left(\frac{\tilde{\eta}}{\eta}-\frac{\tilde{\sigma}}{\sigma}\right) \int_{p^{\prime}} \frac{\boldsymbol{k}^{2}-\left(\boldsymbol{k} \cdot \boldsymbol{k}^{\prime}\right) / \boldsymbol{k}^{\prime 2}}{G\left(p^{\prime}\right) F\left(p^{\prime \prime}\right)} \\
& =\frac{i T \chi \boldsymbol{k}^{2}}{w}\left(\frac{\tilde{\eta}}{\eta}-\frac{\tilde{\sigma}}{\sigma}\right) \mathcal{I}_{0}(p) .
\end{aligned}
$$

Finally, the remaining two momentum fluctuation diagrams in eq. (3.19b) evaluate to

$$
\begin{aligned}
i \Gamma_{a r}^{\prime}(p)= & \frac{2 T \tilde{\eta}}{w} \int_{p^{\prime}} \frac{\boldsymbol{k}^{\prime \prime 2}\left(\lambda_{\pi} \boldsymbol{k}^{2} \delta_{i j}-2 \psi k_{i} k_{j}\right) \bar{k}^{\prime i k} \bar{k}^{\prime \prime j l}\left(\chi_{\epsilon} i \omega^{\prime} \delta_{k l}+\frac{\partial \eta}{\partial \mu}\left(\delta_{k l} \boldsymbol{k}^{\prime} \cdot \boldsymbol{k}^{\prime \prime}+k_{l}^{\prime} k_{k}^{\prime \prime}\right)\right)}{G\left(p^{\prime}\right)\left|G\left(p^{\prime \prime}\right)\right|^{2}} \\
& -T \chi w \tilde{\theta} i \int \frac{\mathrm{d} \omega^{\prime} \mathrm{d}^{d} k^{\prime}}{(2 \pi)^{d+1}} \frac{\left(\lambda_{\pi} \boldsymbol{k}^{2} \delta_{i j}-2 \psi k_{i} k_{j}\right) k^{\prime i k} k^{\prime \prime j l}\left(\delta_{k l} \boldsymbol{k}^{\prime} \cdot \boldsymbol{k}^{\prime \prime}+k_{l}^{\prime} k_{k}^{\prime \prime}\right)}{G\left(p^{\prime}\right) G\left(p^{\prime \prime}\right)} \\
= & i T \int_{p^{\prime}} \frac{\left(\lambda_{\pi} \boldsymbol{k}^{2} \delta_{i j}-2 \psi k_{i} k_{j}\right) \bar{k}^{\prime i k} \bar{k}^{\prime \prime j l}\left(\frac{\tilde{\eta}}{\eta} \chi_{\epsilon} i \omega^{\prime} \delta_{k l}+\left(\frac{\tilde{\eta}}{\eta} \frac{\partial \eta}{\partial \mu}-\frac{\partial \tilde{\eta}}{\partial \mu}\right)\left(\delta_{k l} \boldsymbol{k}^{\prime} \cdot \boldsymbol{k}^{\prime \prime}+k_{l} k_{k}\right)\right)}{G\left(p^{\prime}\right) G\left(p^{\prime \prime}\right)} \\
= & i T \lambda_{\pi} \boldsymbol{k}^{2}\left(\frac{\tilde{\eta}}{w} \chi_{\epsilon}\left(\mathcal{J}_{1}(p)+\frac{1}{2} \mathcal{I}_{3}(0, \boldsymbol{k})\right)+\eta \frac{\partial(\tilde{\eta} / \eta)}{\partial \mu} \mathcal{J}_{2}(p)\right) \\
& -i T \psi \boldsymbol{k}^{2}\left(\frac{\tilde{\eta}}{w} \chi_{\epsilon}\left(\mathcal{J}_{3}(p)+\frac{1}{2} \mathcal{I}_{3}(0, \boldsymbol{k})\right)+\eta \frac{\partial(\tilde{\eta} / \eta)}{\partial \mu} \mathcal{J}_{4}(p)\right) .
\end{aligned}
$$

These expressions can be combined with the ones for $\Gamma_{a r}$ to obtain the retarded correlation function following eq. (B.1).

\section{B.3 Computation of $\Gamma_{a a}$}

We start with the three density fluctuation diagrams in eq. (2.18c), resulting in

$$
\begin{aligned}
\Gamma_{a a}(p) & \sim-2 T^{2} \tilde{\sigma}^{2} \lambda^{2} \boldsymbol{k}^{4} \int_{p^{\prime}} \frac{\boldsymbol{k}^{\prime 2} \boldsymbol{k}^{\prime \prime 2}}{\left|F\left(p^{\prime}\right)\right|^{2}\left|F\left(p^{\prime \prime}\right)\right|^{2}}+\left(4 i T^{2} \chi \lambda \tilde{\lambda} \tilde{\sigma} \int_{p^{\prime}} \frac{\boldsymbol{k}^{\prime 2} \boldsymbol{k}^{2}\left(\boldsymbol{k} \cdot \boldsymbol{k}^{\prime}\right)}{F\left(p^{\prime}\right)\left|F\left(p^{\prime \prime}\right)\right|^{2}}+\text { c.c. }\right) \\
& =T^{2} \chi^{2} \lambda \boldsymbol{k}^{2} \frac{\tilde{\sigma}}{\sigma} \int_{p^{\prime}} \frac{\frac{1}{2} \lambda \tilde{\sigma} / \sigma \boldsymbol{k}^{2}-2 \tilde{\lambda}\left(\boldsymbol{k} \cdot \boldsymbol{k}^{\prime}\right)}{F\left(p^{\prime}\right) F\left(p^{\prime \prime}\right)}+\text { c.c. } \\
& =T^{2} \chi^{2} \lambda \boldsymbol{k}^{2} \frac{\tilde{\sigma}}{\sigma}\left(\frac{\tilde{\sigma}}{\sigma} \lambda-2 \tilde{\lambda}\right) \mathcal{I}_{1}(p)+\text { c.c. }
\end{aligned}
$$


Here "c.c." denotes complex conjugate. Note that the last two diagrams in eq. (2.18c) are complex conjugates of each other. Similarly, we can compute the leading order mixed fluctuation diagram in eq. (3.24c),

$$
\begin{aligned}
\Gamma_{a a}(p) & \sim-\frac{4 T^{2} \tilde{\sigma} \tilde{\eta}}{w^{2}} \int_{p^{\prime}} \frac{\boldsymbol{k}^{\prime 2} \boldsymbol{k}^{\prime \prime 2} \bar{k}^{\prime i j} k_{i} k_{j}}{\left|G\left(p^{\prime}\right)\right|^{2}\left|F\left(p^{\prime \prime}\right)\right|^{2}} \\
& =\frac{T^{2} \chi}{w} \frac{\tilde{\sigma}}{\sigma} \frac{\tilde{\eta}}{\eta} \int_{p^{\prime}} \frac{\boldsymbol{k}^{2}-\left(\boldsymbol{k} \cdot \boldsymbol{k}^{\prime}\right) / \boldsymbol{k}^{\prime 2}}{G\left(p^{\prime}\right) F\left(p^{\prime \prime}\right)}+\text { c.c. } \\
& =\frac{T^{2} \chi \boldsymbol{k}^{2}}{w} \frac{\tilde{\sigma}}{\sigma} \frac{\tilde{\eta}}{\eta} \mathcal{I}_{0}(p)+\text { c.c. }
\end{aligned}
$$

This leaves us with three momentum fluctuation diagrams in eq. (3.24d), which can be computed to obtain

$$
\begin{aligned}
\Gamma_{a a}(p) \sim & -2 T^{2} \tilde{\eta}^{2} \int_{p^{\prime}} \frac{\boldsymbol{k}^{\prime 2} \boldsymbol{k}^{\prime \prime 2}\left(\lambda_{\pi} \delta_{i j} \boldsymbol{k}^{2}-2 \psi k_{i} k_{j}\right) \bar{k}^{\prime i k} \bar{k}^{\prime \prime j l}\left(\lambda_{\pi} \delta_{k l} \boldsymbol{k}^{2}-2 \psi k_{k} k_{l}\right)}{\left|G\left(p^{\prime}\right)\right|^{2}\left|G\left(p^{\prime \prime}\right)\right|^{2}} \\
& -\left(2 i T^{2} w \tilde{\psi} \tilde{\eta} \int_{p^{\prime}} \frac{\boldsymbol{k}^{\prime \prime 2}\left(\lambda_{\pi} \delta_{i j} \boldsymbol{k}^{2}-2 \psi k_{i} k_{j}\right) \bar{k}^{\prime i k} \bar{k}^{\prime \prime j l}\left(\delta_{k l} \boldsymbol{k} \cdot \boldsymbol{k}^{\prime}+k_{k} k_{l}^{\prime}\right)}{G\left(p^{\prime}\right)\left|G\left(p^{\prime \prime}\right)\right|^{2}}+\text { c.c. }\right) \\
= & T^{2} w^{2} \frac{\tilde{\eta}}{\eta} \int_{p^{\prime}} \frac{\left(\lambda_{\pi} \delta_{i j} \boldsymbol{k}^{2}-2 \psi k_{i} k_{j}\right) \bar{k}^{\prime i k} \bar{k}^{\prime \prime j l}\left(\frac{1}{2} \lambda_{\pi} \frac{\tilde{\eta}}{\eta} \delta_{k l} \boldsymbol{k}^{2}+\frac{2 n_{0} \tilde{\eta}}{w^{3}} \delta_{k l} \boldsymbol{k} \cdot \boldsymbol{k}^{\prime}+\frac{n_{0} \tilde{\eta}}{w^{3}} k_{k} k_{l}\right)}{G\left(p^{\prime}\right) G\left(p^{\prime \prime}\right)}+\text { c.c. } \\
= & \frac{T^{2} \tilde{\eta} \boldsymbol{k}^{2}}{w} \frac{\tilde{\eta}}{\eta}\left(\left(\lambda_{\pi} \mathcal{J}_{1}(p)-\psi \mathcal{J}_{3}(p)\right)\left(n_{0}-\chi_{\epsilon} \frac{D \boldsymbol{k}^{2}}{i \omega}\right)+n_{0} \lambda_{\pi} \mathcal{J}_{2}(p)-n_{0} \psi \mathcal{J}_{4}(p)\right)+\text { c.c. }
\end{aligned}
$$

This result can be used in conjunction with $\Gamma_{a r}$ to obtain the symmetric correlation function using eq. (B.3).

Open Access. This article is distributed under the terms of the Creative Commons Attribution License (CC-BY 4.0), which permits any use, distribution and reproduction in any medium, provided the original author(s) and source are credited.

\section{References}

[1] S. Grozdanov and J. Polonyi, Viscosity and dissipative hydrodynamics from effective field theory, Phys. Rev. D 91 (2015) 105031 [arXiv:1305.3670] [InSPIRE].

[2] P. Kovtun, G.D. Moore and P. Romatschke, Towards an effective action for relativistic dissipative hydrodynamics, JHEP 07 (2014) 123 [arXiv:1405.3967] [INSPIRE].

[3] M. Harder, P. Kovtun and A. Ritz, On thermal fluctuations and the generating functional in relativistic hydrodynamics, JHEP 07 (2015) 025 [arXiv: 1502.03076] [INSPIRE].

[4] M. Crossley, P. Glorioso and H. Liu, Effective field theory of dissipative fluids, JHEP 09 (2017) 095 [arXiv: 1511. 03646] [inSPIRE].

[5] F.M. Haehl, R. Loganayagam and M. Rangamani, Topological sigma models $\mathcal{E}$ dissipative hydrodynamics, JHEP 04 (2016) 039 [arXiv: 1511.07809] [INSPIRE]. 
[6] K. Jensen, N. Pinzani-Fokeeva and A. Yarom, Dissipative hydrodynamics in superspace, JHEP 09 (2018) 127 [arXiv: 1701.07436] [INSPIRE].

[7] A. Jain, Effective field theory for non-relativistic hydrodynamics, JHEP 10 (2020) 208 [arXiv: 2008.03994] [INSPIRE].

[8] H. Liu and P. Glorioso, Lectures on non-equilibrium effective field theories and fluctuating hydrodynamics, PoS TASI2017 (2018) 008 [arXiv:1805.09331] [inSPIRE].

[9] A. Jain and P. Kovtun, Non-universality of hydrodynamics, arXiv:2009.01356 [INSPIRE].

[10] X. Chen-Lin, L.V. Delacrétaz and S.A. Hartnoll, Theory of diffusive fluctuations, Phys. Rev. Lett. 122 (2019) 091602 [arXiv: 1811.12540] [INSPIRE].

[11] N. Banerjee, J. Bhattacharya, S. Bhattacharyya, S. Jain, S. Minwalla and T. Sharma, Constraints on Fluid Dynamics from Equilibrium Partition Functions, JHEP 09 (2012) 046 [arXiv: 1203.3544] [INSPIRE].

[12] K. Jensen, M. Kaminski, P. Kovtun, R. Meyer, A. Ritz and A. Yarom, Towards hydrodynamics without an entropy current, Phys. Rev. Lett. 109 (2012) 101601 [arXiv:1203.3556] [INSPIRE].

[13] D. Förster, D.R. Nelson and M.J. Stephen, Long-Time Tails and the Large-Eddy Behavior of a Randomly Stirred Fluid, Phys. Rev. Lett. 36 (1976) 867 [INSPIRE].

[14] R. Kubo, Statistical mechanical theory of irreversible processes. 1. General theory and simple applications in magnetic and conduction problems, J. Phys. Soc. Jap. 12 (1957) 570 [INSPIRE].

[15] P.C. Martin and J.S. Schwinger, Theory of many particle systems. 1., Phys. Rev. 115 (1959) 1342 [INSPIRE].

[16] E. Wang and U.W. Heinz, A generalized fluctuation dissipation theorem for nonlinear response functions, Phys. Rev. D 66 (2002) 025008 [hep-th/9809016] [INSPIRE].

[17] D. Förster, D.R. Nelson and M.J. Stephen, Large-distance and long-time properties of a randomly stirred fluid, Phys. Rev. A 16 (1977) 732 [INSPIRE].

[18] P. Kovtun, Lectures on hydrodynamic fluctuations in relativistic theories, J. Phys. A 45 (2012) 473001 [arXiv: 1205.5040] [INSPIRE].

[19] A. Jain, P. Kovtun, A. Ritz and A. Shukla, Long-time tails in diffusive hydrodynamics, to appear.

[20] O. Narayan and S. Ramaswamy, Anomalous Heat Conduction in One-Dimensional Momentum-Conserving Systems, Phys. Rev. Lett. 89 (2002) 200601 [cond-mat/0205295].

[21] L.D. Landau and E.M. Lifshitz, Hydrodynamic fluctuations, J. Exp. Theor. Phys. 5 (1957) 512 .

[22] P.C. Martin, E.D. Siggia and H.A. Rose, Statistical Dynamics of Classical Systems, Phys. Rev. A 8 (1973) 423 [INSPIRE].

[23] L. Landau, E.M. Lifshitz and L. Pitaevskii, Course of Theoretical Physics: Statistical Physics, Part 2, vol. 9, Butterworth-Heinemann (1980).

[24] P. Kovtun, Fluctuation bounds on charge and heat diffusion, J. Phys. A 48 (2015) 265002 [arXiv: 1407.0690] [INSPIRE]. 
[25] J. Chao and T. Schaefer, Multiplicative noise and the diffusion of conserved densities, JHEP 01 (2021) 071 [arXiv: 2008.01269] [INSPIRE].

[26] D.B. Kaplan, Five lectures on effective field theory, (2005) [nucl-th/0510023] [INSPIRE].

[27] P. Kovtun, G.D. Moore and P. Romatschke, The stickiness of sound: An absolute lower limit on viscosity and the breakdown of second order relativistic hydrodynamics, Phys. Rev. D 84 (2011) 025006 [arXiv: 1104.1586] [INSPIRE]. 